\title{
UNIFORM BMO ESTIMATE OF PARABOLIC EQUATIONS AND GLOBAL WELL-POSEDNESS OF THE THERMISTOR PROBLEM
}

\author{
BUYANG LI $^{1}$ and CHAOXIA YANG ${ }^{2}$ \\ ${ }^{1}$ Department of Mathematics, Nanjing University, Nanjing 210093, PR China; \\ email: buyangli@nju.edu.cn \\ ${ }^{2}$ College of Science, Nanjing University of Posts and Telecommunications, \\ Nanjing 210023, PR China; \\ email: yangcx@njupt.edu.cn
}

Received 4 January 2015; accepted 9 November 2015

\begin{abstract}
We prove global well-posedness of the time-dependent degenerate thermistor problem by establishing a uniform-in-time bounded mean ocsillation (BMO) estimate of inhomogeneous parabolic equations. Applying this estimate to the temperature equation, we derive a BMO bound of the temperature uniform with respect to time, which implies that the electric conductivity is an $A_{2}$ weight. The Hölder continuity of the electric potential is then proved by applying the De GiorgiNash-Moser estimate for degenerate elliptic equations with an $A_{2}$ coefficient. The uniqueness of the solution is proved based on the established regularity of the weak solution. Our results also imply the existence of a global classical solution when the initial and boundary data are smooth.
\end{abstract}

2010 Mathematics Subject Classification: 35K20 (primary); 35Q99 (secondary)

\section{Introduction}

The thermistor problem refers to the heating of a conductor, with temperaturesensitive electric conductivity, by electric current. Let $\phi$ be the electric potential and let $\mathbf{E}=\nabla \phi$ be the electric field. The electric current $\mathbf{J}$ is related to the electric field via $\mathbf{J}=\sigma(u) \mathbf{E}$, where $\sigma(u)$ is the electric conductivity of the conductor, dependent on the temperature $u$. The heat produced (per unit volume) by the electric current is given by Joule's law: $\mathbf{E} \cdot \mathbf{J}=\sigma(u)|\nabla \phi|^{2}$, and the conservation of charge is described by $\nabla \cdot \mathbf{J}=0$.

(c) The Author(s) 2015. This is an Open Access article, distributed under the terms of the Creative Commons Attribution licence (http://creativecommons.org/licenses/by/4.0/), which permits unrestricted re-use, distribution, and reproduction in any medium, provided the original work is properly cited. 
Let $\Omega$ denote the domain possessed by the conductor. Based on the above formulations, the temperature $u$ and the electric potential $\phi$ are governed by the equations

$$
\begin{aligned}
& \frac{\partial u}{\partial t}-\nabla \cdot(\kappa(u) \nabla u)=\sigma(u)|\nabla \phi|^{2}, \\
& -\nabla \cdot(\sigma(u) \nabla \phi)=0,
\end{aligned}
$$

for $x \in \Omega$ and $t>0$, where $\kappa(u)$ is the thermal conductivity. In this paper, we consider the above equations with the Dirichlet boundary/initial conditions:

$$
\begin{array}{ll}
u(x, t)=g(x, t), \quad \phi(x, t)=h(x, t) & \text { for } x \in \partial \Omega \text { and } t>0, \\
u(x, 0)=u_{0}(x) & \text { for } x \in \Omega .
\end{array}
$$

The mathematical expressions of $\sigma(u)$ and $\kappa(u)$ depend on the materials. For some semiconductors, the electric resistivity $\rho(u)=1 / \sigma(u)$ can be approximately expressed as [21]

$$
\rho(u)=\sigma_{0} e^{q / u} u,
$$

and the thermal conductivity $\kappa(u)$ can be regarded as constant (independent of $u$ ). For metallic conductors, the electric conductivity and the thermal conductivity obey the Wiedemann-Franz law [24]:

$$
\frac{\kappa(u)}{\sigma(u)}=L u,
$$

where $L=2.44 \times 10^{-8} \mathrm{~W} \Omega \mathrm{K}^{-2}$ is the Lorenz number. In general, the electric resistivity of metals increases as the temperature rises. At high temperatures, the electric resistivity increases approximately linearly with temperature:

$$
\rho(u)=\rho_{0}\left[1+\alpha\left(u-u_{R}\right)\right]
$$

where $u_{R}$ is some reference temperature and $\alpha$ is called the temperature coefficient of resistivity. If the temperature does not vary much, the above linear formula is often used. More precisely, the electric resistivity is given by the Bloch-Grüneisen formula [33]:

$$
\rho(u)=\rho(0)+a\left(\frac{u}{\Theta}\right)^{m} \int_{0}^{\Theta / u} \frac{s^{m}}{\left(e^{s}-1\right)\left(1-e^{-s}\right)} d s,
$$

where $a, \Theta$ and $m \geqslant 2$ are all positive physical constants.

For both metals and semiconductors, the electric conductivity $\sigma(u)$ tends to zero as the temperature $u$ rises to infinity. The elliptic equation (2) is thus possibly degenerate, which leads to severe difficulties for the analysis of the coupled system. 
The nondegenerate assumption

$$
\sigma_{1} \leqslant \sigma(u) \leqslant \sigma_{2}
$$

is often used to simplify the problem. Mathematical analysis for such a nondegenerate problem has been studied by many authors in the last two decades. The existence of weak solutions was studied by Antontsev and Chipot [5], Allegretto and Xie [3] and Cimatti [8]. With the same nondegenerate assumption, Elliott and Larsson [10] proved the existence of strong solutions for the twodimensional problem by using the energy method (and uniqueness follows). The uniqueness of solutions for the three-dimensional problem is a consequence of the $C^{\alpha}$ regularity proved by Yuan and Liu $[30,31]$ by using the method of layer potentials. Yin [29] proved the same result by using the technique of Campanato spaces. Their results also imply the existence of classical solutions when the boundary and initial data are smooth.

Without the nondegenerate assumption, the problem becomes much more difficult. The partial regularity of the solution was studied by $\mathrm{Xu}$ [27], where the author proved that the solution is smooth in an open subset $D \subset \Omega$ whose complement $\Omega \backslash D$ is of measure zero. Xu [28] also proved the existence of solutions with bounded temperature when the boundary potential is small enough, that is, $\|h\|_{L^{\infty}(\partial \Omega \times(0, T))}$ is small enough. Hachimi and Ammi [14] essentially proved the existence of weak solutions with a truncated nondegenerate electric conductivity. Montesinos and Gallego [22, 23] proved the existence of 'capacity solutions' by considering a new formulation with the transformation $\Phi=\sigma(u) \nabla \phi$. The uniqueness of the weak solution and the existence of global classical solutions remain open. Overall, the main difficulty of the degenerate problem is the lack of an $L^{\infty}$ bound for the temperature $u$.

In this paper, we overcome this difficulty by establishing a uniform-intime BMO estimate for inhomogeneous parabolic equations with possibly discontinuous coefficients. Applying this estimate to the temperature equation, we obtain a uniform-in-time BMO bound of the temperature $u$, as a substitute of the $L^{\infty}$ bound. Based on the BMO bound of the temperature, we further prove that the electric conductivity $\sigma(u)$ is an $A_{2}$ weight uniform in time. The Hölder continuity of the electric potential $\phi$ is then proved by applying the De GiorgiNash-Moser estimate for degenerate elliptic equations with $A_{2}$ coefficient. The Hölder continuity of the temperature is proved by using the Hölder continuity of the electric potential. The existence of a weak solution in a bounded Lipschitz domain is proved, and the uniqueness of the weak solution is proved based on the established regularity of the solution. Our results also imply the existence of a global classical solution when the initial and boundary data are smooth.

For interested readers, we refer to $[2,4,10,12,19,32]$ for numerical methods and numerical analysis of the thermistor problem based on the well-posedness 
assumption. Analyses of the related optimal control problems can be found in $[15,16]$, and the existence of solutions for a related thermoviscoelastic thermistor problem was presented in [18].

The remainder of this paper is organized in the following way. In Section 2 we introduce the notation to be used in this paper and in Section 3 we present our main results. In Section 4, we establish a uniform-in-time BMO estimate for the solutions of inhomogeneous parabolic equations, and in Section 5 we present Hölder estimates of parabolic equations in terms of the Campanato spaces. Based on the estimates obtained in Sections 4 and 5, we prove global existence and uniqueness of a weak solution to the degenerate thermistor problem in Section 6. Conclusions are drawn in Section 7.

\section{Notation}

Before we present our main results, we define the notation to be used in this paper.

Let $n$ be a fixed positive integer and let $B_{R}\left(x_{0}\right)$ denote the ball of radius $R$ centered at the point $x_{0} \in \mathbb{R}^{n}$. Let $\Omega$ be a bounded Lipschitz domain in $\mathbb{R}^{n}$, that is, $\Omega$ is a bounded domain in $\mathbb{R}^{n}$ and for any $y \in \partial \Omega$, there exists a ball $B_{R}(y)$ such that through a rotation of coordinates (if necessary),

$$
B_{R}(y) \cap \Omega=\left\{\left(x_{1}, \ldots, x_{n}\right) \in B_{R}(y): x_{n}>\varphi\left(x_{1}, \ldots, x_{n-1}\right)\right\},
$$

where $\varphi: \mathbb{R}^{n-1} \rightarrow \mathbb{R}$ is a Lipschitz continuous function. For a bounded Lipschitz domain, there exist a positive constant $R_{\Omega}$ and a finite number of balls $B_{R_{\Omega}}\left(y_{1}\right)$, $B_{R_{\Omega}}\left(y_{2}\right), \ldots, B_{R_{\Omega}}\left(y_{m}\right)$ such that $\partial \Omega \subset \bigcup_{j=1}^{m} B_{R_{\Omega} / 2}\left(y_{j}\right)$ and through a rotation of coordinates (if necessary),

$$
B_{2 R_{\Omega}}\left(y_{j}\right) \cap \Omega=\left\{\left(x_{1}, \ldots, x_{n}\right) \in B_{2 R_{\Omega}}\left(y_{j}\right): x_{n}>\varphi_{j}\left(x_{1}, \ldots, x_{n-1}\right)\right\}
$$

for some Lipschitz continuous function $\varphi_{j}: \mathbb{R}^{n-1} \rightarrow \mathbb{R}$.

For any integer $m \geqslant 0,1 \leqslant p \leqslant \infty$ and $0<\alpha<1$, let $W^{m, p}(\Omega)$ and $C^{m+\alpha}(\bar{\Omega})$ denote the usual Sobolev space and Hölder space [1], respectively, and let $C^{m+\alpha}(\Omega)$ denote the space of functions that belong to $C^{m+\alpha}(\bar{B})$ for any closed ball $\bar{B} \subset \Omega$. Let $C_{0}^{m+\alpha}(\bar{\Omega})$ be the subspace of $C^{m+\alpha}(\bar{\Omega})$ consisting of functions vanishing on the boundary $\partial \Omega$.

Let $|D|$ denote the Lebesgue measure for any measurable subset $D$ of $\mathbb{R}^{n}$, and let $B_{R}\left(x_{0}\right)$ denote the ball of radius $R$ centered at the point $x_{0} \in \mathbb{R}^{n}$. We say that a positive locally integrable function $w$ defined on $\mathbb{R}^{n}$ is an $A_{2}$ weight if

$$
\sup _{B \subset \mathbb{R}^{n}}\left(\frac{1}{|B|} \int_{B} w(x) d x\right)\left(\frac{1}{|B|} \int_{B} \frac{1}{w(x)} d x\right) \leqslant C
$$

for some positive constant $C$, where the supremum extends over all balls in $B$ in $\mathbb{R}^{n}$. 
For any measurable subset $D$ of $\mathbb{R}^{n}$, we let $f_{D}=(1 /|D|) \int_{D} f(x) d x$ denote the average of $f$ over $D$. For $1 \leqslant p<\infty$ and $0 \leqslant \theta \leqslant 1$, let $L^{p, \theta}(\Omega)$ denote the Morrey space of measurable functions $f$ such that

$$
\|f\|_{L^{p, \theta}(\Omega)}:=\sup _{B_{R}\left(x_{0}\right)}\left(\frac{1}{R^{n \theta}} \int_{B_{R}\left(x_{0}\right) \cap \Omega}|f(x)|^{p} d x\right)^{1 / p}<\infty,
$$

where the supremum above extends over all balls $B_{R}\left(x_{0}\right)$ with $x_{0} \in \bar{\Omega}$ and $0<R<R_{\Omega}$. For $1 \leqslant p<\infty$ and $1 \leqslant \theta<\infty$, let $\mathcal{L}^{p, \theta}(\Omega)$ denote the Campanato space of functions bounded (or vanishing for $\theta>1$ ) on the boundary $\partial \Omega$, equipped with the norm

$$
\begin{aligned}
\|f\|_{\mathcal{L}^{p, \theta}(\Omega)}:= & \sup _{B_{R}\left(x_{0}\right) \cap \Omega}\left(\frac{1}{R^{n \theta}} \int_{B_{R}\left(x_{0}\right) \cap \Omega}|f(x)|^{p} d x\right)^{1 / p} \\
& +\sup _{B_{R}\left(y_{0}\right) \cap \Omega}\left(\frac{1}{R^{n \theta}} \int_{B_{R}\left(y_{0}\right) \cap \Omega}\left|f(x)-f_{B_{R}\left(y_{0}\right) \cap \Omega}\right|^{p} d x\right)^{1 / p},
\end{aligned}
$$

where the supremum above extends over all balls with $x_{0} \in \partial \Omega, y_{0} \in \Omega$ and $0<R<R_{\Omega}$, and we set $\overline{\mathrm{BMO}}=\mathcal{L}^{1,1}(\Omega)$.

For any fixed $T>0$, we set $\Omega_{T}=\Omega \times(0, T]$ and $\Gamma_{T}=\partial \Omega \times(0, T]$. For any point $\left(x_{0}, t_{0}\right) \in \mathbb{R}^{n+1}$, we set $Q_{R}\left(x_{0}, t_{0}\right)=B_{R}\left(x_{0}\right) \times\left(t_{0}-R^{2}, t_{0}\right]$ as the parabolic cylinder centered at $\left(x_{0}, t_{0}\right)$ of radius $R$. For integers $m, n \geqslant 0,0<\alpha, \beta<1$ and any open subset $Q \subset \Omega_{T}$, let $C^{m+\alpha, n+\beta}(\bar{Q})$ denote the anisotropic Hölder space of functions, equipped with the norm $\|f\|_{C^{m+\alpha, n+\beta}(\bar{Q})}:=\|f\|_{L^{\infty}(Q)}+|f|_{C^{m+\alpha, n+\beta}(\bar{Q})}$, where

$$
\begin{aligned}
|f|_{C^{m+\alpha, n+\beta}(\bar{Q})}= & \sum_{|\gamma|=m} \sup _{\substack{(x, t) \in Q \\
(y, s) \in Q}} \frac{\left|D_{x}^{\gamma} f(x, t)-D_{x}^{\gamma} f(y, s)\right|}{|x-y|^{\alpha}+|t-s|^{\beta}} \\
& +\sup _{\substack{(x, t) \in Q \\
(y, s) \in Q}} \frac{\left|D_{t}^{n} f(x, t)-D_{t}^{n} f(y, s)\right|}{|x-y|^{\alpha}+|t-s|^{\beta}},
\end{aligned}
$$

and set $C^{\alpha}\left(\bar{\Omega}_{T}\right)=C^{\alpha, \alpha}\left(\bar{\Omega}_{T}\right)$. Let $C_{0}^{m+\alpha, n+\beta}(\bar{Q})$ denote the subspace of $C^{m+\alpha, n+\beta}(\bar{Q})$ with functions vanishing on the boundary $\partial \Omega$. Let $C^{\infty}(\bar{Q})$ denote the space of functions whose partial derivatives up to all orders are uniformly continuous on $\bar{Q}$. Let $C^{m+\alpha, n+\beta}\left(\Omega_{T}\right)$ and $C^{\infty}\left(\Omega_{T}\right)$ denote the space of functions that are in $C^{m+\alpha, n+\beta}(\bar{Q})$ and $C^{\infty}(\bar{Q})$ for any closed cylinder $\bar{Q} \subset \Omega_{T}$, respectively. For any measurable subset $Q$ of $\mathbb{R}^{n+1}$ and any integrable function $f$ defined on $Q$, we let $|Q|$ denote the Lebesgue measure of $Q$ and let $f_{Q}=(1 /|Q|) \int_{Q} f(x) d x$ denote the average of $f$ over $Q$. Analogous to the 
Morrey space $L^{p, \theta}(\Omega)$ and the Campanato space $\mathcal{L}^{p, \theta}(\Omega)$, for $1 \leqslant p<\infty$ we can define the parabolic Morrey space $L_{\text {para }}^{p, \theta}\left(\Omega_{T}\right)$ equipped with the norm

$$
\|f\|_{L_{\text {para }}^{p, \theta}\left(\Omega_{T}\right)}=\sup _{Q_{R}}\left(\frac{1}{R^{(n+2) \theta}} \int_{Q_{R}}|f(x, t)|^{p} d x d t\right)^{1 / p}, \quad 0 \leqslant \theta \leqslant 1,
$$

and the parabolic Campanato space $\mathcal{L}_{\text {para }}^{p, \theta}\left(\Omega_{T}\right)$ of functions vanishing on the boundary $\Gamma_{T}$, equipped with the norm

$$
\begin{aligned}
\|f\|_{\mathcal{L}_{\text {para }}^{p, \theta}\left(\Omega_{T}\right)}:= & \sup _{Q_{R}\left(x_{0}, t_{0}\right) \cap \Omega_{T}}\left(\frac{1}{R^{(n+2) \theta}} \int_{Q_{R}\left(x_{0}, t_{0}\right) \cap \Omega_{T}}|f(x, t)|^{p} d x d t\right)^{1 / p}, \\
& +\sup _{Q_{R}\left(y_{0}, s_{0}\right) \cap \Omega_{T}}\left(\frac{1}{R^{(n+2) \theta}} \int_{Q_{R}\left(y_{0}, s_{0}\right) \cap \Omega_{T}}\left|f(x, t)-f_{Q_{R}}\right|^{p} d x d t\right)^{1 / p},
\end{aligned}
$$

where the suprema above extend over all cylinders with $x_{0} \in \partial \Omega, y_{0} \in \Omega, t_{0}$, $s_{0} \in(0, T]$ and $0<R<R_{\Omega}$.

For any Banach space $X$ and time interval $\left(t_{1}, t_{2}\right) \subset \mathbb{R}$, we denote by $L^{p}\left(\left(t_{1}, t_{2}\right)\right.$; $X)$ the Bochner space equipped with the norm

$$
\|f\|_{L^{p}\left(\left(t_{1}, t_{2}\right) ; X\right)}= \begin{cases}\left(\int_{t_{1}}^{t_{2}}\|f(t)\|_{X}^{p} d t\right)^{1 / p}, & 1 \leqslant p<\infty, \\ \operatorname{ess~sup~}_{t \in\left(t_{1}, t_{2}\right)}\|f(t)\|_{X}, & p=\infty .\end{cases}
$$

The importance of the (parabolic) Morrey spaces is that $L^{p, \theta}(\Omega)$ rescales just like $L^{p /(1-\theta)}(\Omega)$, that is, through the transformation $\tilde{f}(y)=f(R y)$ we have

$$
\|f\|_{L^{p, \theta}\left(B_{R}\right)}=R^{n(1-\theta) / p}\|\tilde{f}\|_{L^{p, \theta}\left(B_{1}\right)}
$$

just like

$$
\|f\|_{L^{p /(1-\theta)}\left(B_{R}\right)}=R^{n(1-\theta) / p}\|\tilde{f}\|_{L^{p /(1-\theta)}\left(B_{1}\right)},
$$

for any ball $B_{R} \subset \Omega$. Similarly, $L_{\text {para }}^{p, \theta}\left(\Omega_{T}\right)$ rescales just like $L^{p /(1-\theta)}\left(\Omega_{T}\right)$. Therefore, $L^{p, \theta}(\Omega)$ and $L_{\text {para }}^{p, \theta}\left(\Omega_{T}\right)$ can be used as substitute for $L^{p /(1-\theta)}(\Omega)$ and $L^{p /(1-\theta)}\left(\Omega_{T}\right)$, respectively, with lower order integrability. The importance of the (parabolic) Campanato spaces includes:

(1) $\mathcal{L}^{p, 1}(\Omega)$ are equivalent for all $1 \leqslant p<\infty$, that is, $\mathcal{L}^{p, 1}(\Omega) \cong \overline{\mathrm{BMO}}$.

(2) If $1<\theta<(n+p) / n$, then $\mathcal{L}^{p, \theta}(\Omega) \cong C_{0}^{\alpha}(\bar{\Omega})$ for $\alpha=n(\theta-1) / p$.

(3) If $1<\theta<(n+2+p) /(n+2)$, then $\mathcal{L}_{\text {para }}^{p, \theta}\left(\Omega_{T}\right) \cong C_{0}^{\alpha, \alpha / 2}\left(\bar{\Omega}_{T}\right)$ for $\alpha=$ $(n+2)(\theta-1) / p$.

These properties of the Morrey and Campanato spaces can be found in [7, 25]. 
In this paper, we let $C_{p_{1}, p_{2}, \ldots, p_{m}}$ denote a generic positive constant, which depends on the parameters $p_{1}, p_{2}, \ldots, p_{m}$.

\section{Main results}

First, we establish a uniform-in-time BMO estimate and a Hölder estimate for the solution of the parabolic equation

$$
\begin{cases}\frac{\partial u}{\partial t}-\nabla \cdot(A \nabla u)=\nabla \cdot \vec{f}+f_{0} & \text { in } \Omega \times(0, T), \\ u=g & \text { on } \partial \Omega \times(0, T), \\ u(x, 0)=u_{0}(x) & \text { for } x \in \Omega,\end{cases}
$$

where $A(x, t)=\left[A_{i j}(x, t)\right]_{n \times n}$ is a symmetric positive definite measurable matrix function defined on $\mathbb{R}^{n+1}$ such that

$$
K^{-1}|\xi|^{2} \leqslant \sum_{i, j=1}^{n} A_{i j}(x, t) \xi_{i} \xi_{j} \leqslant K|\xi|^{2} \quad \text { for all } \xi \in \mathbb{R}^{n}
$$

holds almost everywhere for $(x, t) \in \mathbb{R}^{n+1}$, where $K$ is a positive constant.

THEOREM 1 (BMO and Hölder estimates of parabolic equations). There exist positive constants $C$ and $\alpha_{0} \in(0,1)$ depending only on the elliptic constant $K$, the domain $\Omega$ and the dimension $n$ (independent of $T$ ), such that the solution of (4) satisfies the BMO estimate

$$
\|u\|_{L^{\infty}((0, T) ; \overline{\mathrm{BMO}})} \leqslant C\left(\left\|f_{0}\right\|_{L^{1, n /(n+2)}\left(\Omega_{T}\right)}+\|\vec{f}\|_{L^{2, n /(n+2)}\left(\Omega_{T}\right)}+\left\|u_{0}\right\|_{L^{\infty}(\Omega)}+\|g\|_{L^{\infty}\left(\Gamma_{T}\right)}\right) .
$$

If the compatibility condition $u_{0}(x)=g(x, 0)$ for $x \in \partial \Omega$ is satisfied, then we have

$$
\begin{aligned}
\|u\|_{C^{\alpha, \alpha / 2}\left(\bar{\Omega}_{T}\right)} \leqslant & C\left(\left\|f_{0}\right\|_{L^{1,(n+\alpha) /(n+2)}\left(\Omega_{T}\right)}+\|\vec{f}\|_{L^{2,(n+2 \alpha) /(n+2)\left(\Omega_{T}\right)}}\right. \\
& \left.+\left\|u_{0}\right\|_{C^{\alpha}(\bar{\Omega})}+\|g\|_{C^{\alpha, \alpha / 2}\left(\Gamma_{T}\right)}\right),
\end{aligned}
$$

for $0<\alpha \leqslant \alpha_{0}$.

Inequality (6) is new. An inequality similar to (7) was proved in [29],

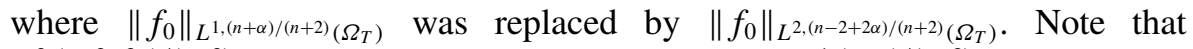
$L^{2,(n-2+2 \alpha) /(n+2)}\left(\Omega_{T}\right)$ rescales in the same way as $L^{1,(n+\alpha) /(n+2)}\left(\Omega_{T}\right)$ under a scale transformation but requires higher integrability.

Second, by applying Theorem 1, we prove global existence and uniqueness of a weak solution for the degenerate thermistor problem under the following physical hypotheses: 
(H1) The thermal conductivity is a smooth function of temperature and satisfies

$$
0<\inf _{s \geqslant r} \kappa(s) \leqslant \sup _{s \geqslant r} \kappa(s)<\infty \quad \text { for any fixed } r>0 .
$$

(H2) The electric resistivity $\rho(u)=1 / \sigma(u)$ is a smooth function of temperature such that for some $p>0$ there holds

$$
C_{1, r}+C_{2, r} s^{p} \leqslant \rho(s) \leqslant C_{3, r}+C_{4, r} s^{p} \quad \forall s \geqslant r>0,
$$

where $C_{i, r}, i=1, \ldots, 5$, are some positive constants (possibly depending on $r$ ).

Clearly, hypotheses $(\mathrm{H} 1)$ and $(\mathrm{H} 2)$ are true for metals and some semiconductors. In particular, the electric resistivity $\rho(u)$ can be any polynomial that is positive for $u>0$. Physical hypotheses (H1) and (H2) also imply that for any given $r>0, \sigma(s)$ is bounded for $s \geqslant r$.

THEOREM 2 (Global well-posedness of the degenerate thermistor problem). Let $\Omega$ be a bounded Lipschitz domain in $\mathbb{R}^{n}(n=2,3)$ and let $q_{0}>n$. Assume that $u_{0} \in W^{1, q_{0}}(\Omega), g \in L^{\infty}\left((0, T) ; W^{1, q_{0}}(\Omega)\right), \partial_{t} g \in L^{\infty}\left((0, T) ; L^{q_{0}}(\Omega)\right)$, $h \in L^{\infty}\left((0, T) ; W^{1, q_{0}}(\Omega)\right)$, with

$$
\min _{(x, t) \in \Gamma_{T}} g(x, t)>0, \quad \min _{x \in \Omega} u_{0}(x)>0,
$$

and $g(x, 0)=u_{0}(x)$ for $x \in \partial \Omega$. Then, under hypotheses $(\mathrm{H} 1)$ and $(\mathrm{H} 2)$, the initial-boundary value problem (1)-(3) admits a unique weak solution $(u, \phi)$ such that

$$
\begin{aligned}
& u \in C^{\alpha, \alpha / 2}\left(\bar{\Omega}_{T}\right) \cap L^{p}\left((0, T) ; W^{1, q}(\Omega)\right), \quad \phi \in L^{\infty}\left((0, T) ; W^{1, q}(\Omega)\right), \\
& \partial_{t} u \in L^{p}\left((0, T) ; W^{-1, q}(\Omega)\right),
\end{aligned}
$$

for some $q>n, 0<\alpha<1$ and any $1<p<\infty$, in the sense that the equations

$$
\begin{aligned}
& \int_{0}^{T} \int_{\Omega} \frac{\partial u}{\partial t} v d x d t+\int_{0}^{T} \int_{\Omega} \kappa(u) \nabla u \cdot \nabla v d x d t=\int_{0}^{T} \int_{\Omega} \sigma(u)|\nabla \phi|^{2} v d x d t, \\
& \int_{0}^{T} \int_{\Omega} \sigma(u) \nabla \phi \cdot \nabla \varphi d x d t=0,
\end{aligned}
$$

hold for any $v, \varphi \in L^{2}\left((0, T) ; H_{0}^{1}(\Omega)\right)$.

Note that with the regularity (9), the last equation above is equivalent to

$$
\int_{\Omega} \sigma(u) \nabla \phi \cdot \nabla \varphi d x=0, \quad \forall \varphi \in H_{0}^{1}(\Omega), \quad \text { a.e. } t \in(0, T) .
$$




\section{BMO estimate of parabolic equations}

The solution of (4) can be decomposed into three parts, that is, the solution of the following three problems:

$$
\begin{aligned}
& \left\{\begin{array}{lc}
\frac{\partial u}{\partial t}-\nabla \cdot(A \nabla u)=f_{0}, & \text { in } \Omega \times(0, T), \\
u=0 & \text { on } \partial \Omega \times(0, T), \\
u(x, 0)=0 & \text { for } x \in \Omega .
\end{array}\right. \\
& \left\{\begin{array}{lc}
\frac{\partial u}{\partial t}-\nabla \cdot(A \nabla u)=\nabla \cdot \vec{f}, & \text { in } \Omega \times(0, T), \\
u=0 & \text { on } \partial \Omega \times(0, T), \\
u(x, 0)=0 & \text { for } x \in \Omega,
\end{array}\right. \\
& \left\{\begin{array}{lc}
\frac{\partial u}{\partial t}-\nabla \cdot(A \nabla u)=0, & \text { in } \Omega \times(0, T), \\
u=g & \text { on } \partial \Omega \times(0, T), \\
u(x, 0)=u_{0}(x) & \text { for } x \in \Omega .
\end{array}\right.
\end{aligned}
$$

From the maximum principle and the De Giorgi-Nash-Moser estimates, we know that there exist positive constants $C$ and $0<\alpha_{0}<1$ such that the solution of (12) satisfies

$$
\begin{aligned}
& \|u\|_{L^{\infty}\left(\Omega_{T}\right)} \leqslant\|g\|_{L^{\infty}\left(\Gamma_{T}\right)}+\left\|u_{0}\right\|_{L^{\infty}(\Omega)}, \\
& \|u\|_{C^{\alpha, \alpha / 2}\left(\bar{\Omega}_{T}\right)} \leqslant C\left(\|g\|_{C^{\alpha, \alpha / 2}\left(\bar{\Gamma}_{T}\right)}+\left\|u_{0}\right\|_{C^{\alpha}(\bar{\Omega})}\right),
\end{aligned}
$$

for $0<\alpha<\alpha_{0}<1$ and $T>0$ (the second inequality above requires the compatibility condition). To prove Theorem 1 , it suffices to present estimates for Equations (10) and (11).

The remainder of this section is organized in the following way. In Section 4.1, we present local $L^{1}$ estimates for the solution to (10). In Section 4.2, we combine the local $L^{1}$ estimates to derive a global BMO estimate based on the equivalence of $\overline{\mathrm{BMO}}$ with the Campanato space $\mathcal{L}^{1,1}(\Omega)$. In Section 4.3 , we establish the BMO estimate for (11) in terms of the Campanato space $\mathcal{L}^{2,1}(\Omega)$.

4.1. Local $L^{\mathbf{1}}$ estimates. In this subsection, we present local $L^{1}$ estimates for the solution of (10). The estimates obtained in this subsection will be used in Section 4.2 to derive a global BMO estimate uniformly with respect to time.

Lemma 3. Let $x_{0} \in \Omega$ and $0<t_{0}<T$. There exist $\alpha_{0} \in(0,1)$ and $C>0$ such that if $u$ is the solution of (10) in $Q_{R}=B_{R}\left(x_{0}\right) \times I_{R}$ with $I_{R}=\left(t_{0}-R^{2}, t_{0}\right]$, 
then

$$
\max _{t \in I_{\rho}}\left\|u-u_{Q_{\rho}}\right\|_{L^{1}\left(B_{\rho}\right)} \leqslant C\left(\frac{\rho}{R}\right)^{n+\alpha_{0}} \max _{t \in I_{R}}\|u-\theta\|_{L^{1}\left(B_{R}\right)}+C\left\|f_{0}\right\|_{L^{1}\left(Q_{R}\right)}
$$

holds for all $0<\rho \leqslant R \leqslant \min \left(\operatorname{dist}\left(x_{0}, \partial \Omega\right), \sqrt{t_{0}}\right)$, where the constants $C$ and $\alpha_{0}$ depend only on $K$ and $n$.

Proof. First, we prove the lemma for $\theta=0$. Let $\widetilde{B}_{r}=B_{r}(0), \widetilde{I}_{r}=\left(-r^{2}, 0\right]$ and $\widetilde{\Gamma}_{r}=\partial \widetilde{B}_{r} \times \widetilde{I}_{r}$. With any function $\xi$ defined on $Q_{R}$, we associate a function $\tilde{\xi}(y, s)=\xi\left(x_{0}+R y, t_{0}+R^{2} s\right)$ defined on $\widetilde{Q}_{1}:=\widetilde{B}_{1} \times \widetilde{I}_{1}$. Then $\tilde{u}$ is a solution to the equation

$$
\frac{\partial \tilde{u}}{\partial s}-\nabla_{y} \cdot\left(\tilde{A} \nabla_{y} \tilde{u}\right)=R^{2} \tilde{f}_{0}
$$

in $\widetilde{Q}_{1}$. Let $w$ be the solution of

$$
\frac{\partial w}{\partial s}-\nabla_{y} \cdot\left(\tilde{A} \nabla_{y} w\right)=R^{2} \tilde{f}_{0}
$$

with the boundary/initial condition $w=0$ on the parabolic boundary $\partial_{\mathrm{p}} \widetilde{Q}_{1}$ and let $\bar{w}$ be the solution of

$$
\frac{\partial \bar{w}}{\partial s}-\nabla_{y} \cdot\left(\tilde{A} \nabla_{y} \bar{w}\right)=R^{2}\left|\tilde{f}_{0}\right| 1_{\widetilde{Q}_{1}}
$$

in $\mathbb{R}^{n+1}$ with the initial condition $\bar{w}(y,-1) \equiv 0$. By the maximum principle, we know that

$$
\begin{aligned}
|w(y, s)| & \leqslant|\bar{w}(y, s)| \\
& \leqslant \int_{-1}^{s} \int_{\mathbb{R}^{n}} \frac{C}{\left(s-s^{\prime}\right)^{n / 2}} e^{-\left(\left|y-y^{\prime}\right|^{2}\right) /\left(C\left(s-s^{\prime}\right)\right)} R^{2}\left|\tilde{f}_{0}\left(y^{\prime}, s^{\prime}\right)\right| 1_{\widetilde{Q}_{1}}\left(y^{\prime}, s^{\prime}\right) d y^{\prime} d s^{\prime} .
\end{aligned}
$$

Taking the $L^{1}\left(\widetilde{B}_{1}\right)$ norm with respect to $y$, we derive

$$
\|w\|_{L^{\infty}\left(\widetilde{I}_{1} ; L^{1}\left(\widetilde{B}_{1}\right)\right)} \leqslant C R^{2}\left\|\tilde{f}_{0}\right\|_{L^{1}\left(\widetilde{Q}_{1}\right)} .
$$

We note that $v=\tilde{u}-\tilde{u}_{\widetilde{Q}_{1}}-w$ is the solution of

$$
\frac{\partial v}{\partial s}-\nabla_{y} \cdot\left(\tilde{A} \nabla_{y} v\right)=0
$$

in $\widetilde{Q}_{1}$, and by the De Giorgi-Nash estimates of parabolic equations [20, Theorems 6.28 and 6.17], with $k_{1}=k_{2}=k=0$ ) we know that there exists $\alpha_{0} \in(0,1)$ such that for $\rho \in(0,1 / 2]$,

$\max _{t \in \widetilde{I}_{\rho}} \frac{1}{\rho^{n+\alpha_{0}}} \int_{\widetilde{B}_{\rho}}\left|v-v \widetilde{Q}_{\rho}\right| d y \leqslant C|v|_{C^{\alpha_{0}, \alpha_{0} / 2}\left(\widetilde{Q}_{1 / 2}\right)} \leqslant C\|v\|_{L^{1}\left(\widetilde{Q}_{1}\right)} \leqslant C \max _{t \in \widetilde{I}_{1}}\|v\|_{L^{1}\left(\widetilde{B}_{1}\right)}$. 
Therefore,

$$
\begin{aligned}
\max _{t \in \widetilde{I}_{\rho}}\left\|\tilde{u}-\tilde{u}_{\widetilde{Q}_{\rho}}\right\|_{L^{1}\left(\widetilde{B}_{\rho}\right)} & \leqslant \max _{t \in \widetilde{I}_{\rho}}\left\|v-v \widetilde{Q}_{\rho}\right\|_{L^{1}\left(\widetilde{B}_{\rho}\right)}+\max _{t \in \widetilde{I}_{\rho}}\left\|w-w_{\widetilde{Q}_{\rho}}\right\|_{L^{1}\left(\widetilde{B}_{\rho}\right)} \\
& \leqslant C \rho^{n+\alpha_{0}} \max _{t \in \widetilde{I}_{1}}\|v\|_{L^{1}\left(\widetilde{B}_{1}\right)}+C \max _{t \in \widetilde{I}_{1}}\|w\|_{L^{1}\left(\widetilde{B}_{1}\right)} \\
& \leqslant C \rho^{n+\alpha_{0}} \max _{t \in \widetilde{I}_{1}}\left\|\tilde{u}-\tilde{u}_{\widetilde{Q}_{1}}\right\|_{L^{1}\left(\widetilde{B}_{1}\right)}+C \max _{t \in \widetilde{I}_{1}}\|w\|_{L^{1}\left(\widetilde{B}_{1}\right)} \\
& \leqslant C \rho^{n+\alpha_{0}} \max _{t \in \widetilde{I}_{1}}\left\|\tilde{u}-\tilde{u}_{\widetilde{Q}_{1}}\right\|_{L^{1}\left(\widetilde{B}_{1}\right)}+C R^{2}\left\|\tilde{f}_{0}\right\|_{L^{1}\left(\widetilde{Q}_{1}\right)} \\
& \leqslant C \rho^{n+\alpha_{0}} \max _{t \in \widetilde{I}_{1}}\|\tilde{u}\|_{L^{1}\left(\widetilde{B}_{1}\right)}+C R^{2}\left\|\tilde{f}_{0}\right\|_{L^{1}\left(\widetilde{Q}_{1}\right)},
\end{aligned}
$$

where we have noted that

$$
\left\|\widetilde{u} \widetilde{Q}_{1}\right\|_{L^{1}\left(\widetilde{B}_{1}\right)}=\frac{\left|\widetilde{B}_{1}\right|}{\left|\widetilde{Q}_{1}\right|} \int_{\widetilde{Q}_{1}}|\widetilde{u}| d x d t \leqslant \max _{t \in \widetilde{L}_{1}}\|\widetilde{u}\|_{L^{1}\left(\widetilde{B}_{1}\right)} .
$$

Transforming back to the $(x, t)$-coordinates, we complete the proof of the lemma for $\theta=0$.

Then we note that $u-\theta$ is also a solution to Equation (10) in $Q_{R}$ for any $\theta \in \mathbb{R}$.

Similarly, we can prove the following local $L^{1}$ estimates near the boundary $\partial_{p} \Omega_{T}$.

LEMmA 4. Let $x_{0} \in \Omega$ and $t_{0}=0$. There exist $\alpha_{0} \in(0,1)$ and $C>0$ such that if $u$ is the solution of $(10)$ in $Q_{R}=B_{R}\left(x_{0}\right) \times \underline{I}_{R}$ with $\underline{I}_{R}=\left[0, R^{2}\right]$, then

$$
\max _{t \in \underline{I}_{\rho}}\|u\|_{L^{1}\left(B_{\rho}\right)} \leqslant C\left(\frac{\rho}{R}\right)^{n+\alpha_{0}} \max _{t \in \underline{I}_{R}}\|u\|_{L^{1}\left(B_{R}\right)}+C\left\|f_{0}\right\|_{L^{1}\left(Q_{R}\right)}
$$

holds for any $0<\rho \leqslant R \leqslant \min \left(\operatorname{dist}\left(x_{0}, \partial \Omega\right), \sqrt{T}\right)$, where the constants $C$ and $\alpha_{0}$ depend only on $K$ and $n$.

LeMmA 5. Let $x_{0} \in \partial \Omega$ and $t_{0}>0$. There exist $\alpha_{0} \in(0,1)$ and $C>0$ such that if $u$ is the solution of (10) in $Q_{R}=B_{R} \times I_{R}$, with $B_{R}=B_{R}\left(x_{0}\right) \cap \Omega$ and $I_{R}=\left(t_{0}-R^{2}, t_{0}\right]$, then

$$
\max _{t \in I_{\rho}}\|u\|_{L^{1}\left(B_{\rho}\right)} \leqslant C\left(\frac{\rho}{R}\right)^{n+\alpha_{0}} \max _{t \in I_{R}}\|u\|_{L^{1}\left(B_{R}\right)}+C\left\|f_{0}\right\|_{L^{1}\left(Q_{R}\right)}
$$

holds for any $0<\rho \leqslant R \leqslant \min \left(R_{\Omega}, \sqrt{t_{0}}\right)$, where the constants $C$ and $\alpha_{0}$ depend only on $K, n$ and $\Omega$. 
LemMA 6. Let $x_{0} \in \partial \Omega$ and $t_{0}=0$. There exist $\alpha_{0} \in(0,1)$ and $C>0$ such that if $u$ is the solution of (10) in $Q_{R}=B_{R} \times I_{R}$, with $B_{R}=B_{R}\left(x_{0}\right) \cap \Omega$ and $I_{R}=\left[0, R^{2}\right]$, then

$$
\max _{t \in I_{\rho}}\|u\|_{L^{1}\left(B_{\rho}\right)} \leqslant C\left(\frac{\rho}{R}\right)^{n+\alpha_{0}} \max _{t \in I_{R}}\|u\|_{L^{1}\left(B_{R}\right)}+C\left\|f_{0}\right\|_{L^{1}\left(Q_{R}\right)}
$$

holds for any $0<\rho \leqslant R \leqslant \min \left(R_{\Omega}, \sqrt{T}\right)$, where the constants $C$ and $\alpha_{0}$ depend only on $K, n$ and $\Omega$.

The following simple lemma can be found in [7, 20], which has been widely used in the analysis of elliptic and parabolic equations in terms of the Morrey and Campanato spaces.

LEMMA 7. Let $\varphi(\cdot)$ be a nonnegative and nondecreasing function defined on $\left(0, R_{0}\right]$ and suppose that for any $0<\rho \leqslant R \leqslant R_{0}$,

$$
\varphi(\rho) \leqslant C_{1}\left(\frac{\rho}{R}\right)^{\gamma_{1}} \varphi(R)+C_{2} R^{\gamma_{2}},
$$

where $C_{1}, \gamma_{1}$ and $\gamma_{2}$ are nonnegative constants such that $0<\gamma_{2}<\gamma_{1}$. Then

$$
\frac{1}{R^{\gamma_{2}}} \varphi(R) \leqslant C_{\gamma_{1}, \gamma_{2}, C_{1}}\left(\frac{1}{R_{0}^{\gamma_{2}}} \varphi\left(R_{0}\right)+C_{2}\right) .
$$

By applying Lemma 7 to Lemmas 3-6, we obtain the following local $L^{1}$ estimates.

Proposition 8. For $x_{0} \in \Omega, t_{0}>0$ and $Q_{R}=B_{R}\left(x_{0}\right) \times I_{R}$ with $I_{R}=\left(t_{0}-R^{2}\right.$, $\left.t_{0}\right]$, we have

$$
\begin{aligned}
& \frac{1}{\rho^{n}}\left\|u-u_{Q_{\rho}}\right\|_{L^{\infty}\left(\left(t_{0}-\rho^{2}, t_{0}\right) ; L^{1}\left(B_{\rho}\right)\right)} \\
& \quad \leqslant C\left(\frac{1}{R^{n}}\|u\|_{L^{\infty}\left(\left(t_{0}-R^{2}, t_{0}\right) ; L^{1}\left(B_{R}\right)\right)}+\left\|f_{0}\right\|_{L^{1, n /(n+2)}\left(\Omega_{T}\right)}\right)
\end{aligned}
$$

for any $0<\rho \leqslant R \leqslant \min \left(\operatorname{dist}\left(x_{0}, \partial \Omega\right), \sqrt{t_{0}}\right)$.

Proposition 9. For $x_{0} \in \Omega, t_{0}=0$ and $Q_{R}=B_{R}\left(x_{0}\right) \times\left[0, R^{2}\right]$, we have

$$
\frac{1}{\rho^{n}}\|u\|_{L^{\infty}\left(\left(t_{0}-\rho^{2}, t_{0}\right) ; L^{1}\left(B_{\rho}\right)\right)} \leqslant C\left(\frac{1}{R^{n}}\|u\|_{L^{\infty}\left(\left(t_{0}-R^{2}, t_{0}\right) ; L^{1}\left(B_{R}\right)\right)}+\left\|f_{0}\right\|_{L^{1, n /(n+2)}\left(\Omega_{T}\right)}\right)
$$

for any $0<\rho \leqslant R \leqslant \min \left(\operatorname{dist}\left(x_{0}, \partial \Omega\right), \sqrt{T}\right)$. 
Proposition 10. For $x_{0} \in \partial \Omega, t_{0}>0$ and $Q_{R}=B_{R}\left(x_{0}\right) \cap \Omega \times I_{R}$ with $I_{R}=$ $\left(t_{0}-R^{2}, t_{0}\right]$, we have

$$
\frac{1}{\rho^{n}}\|u\|_{L^{\infty}\left(\left(t_{0}-\rho^{2}, t_{0}\right) ; L^{1}\left(B_{\rho}\right)\right)} \leqslant C\left(\frac{1}{R^{n}}\|u\|_{L^{\infty}\left(\left(t_{0}-R^{2}, t_{0}\right) ; L^{1}\left(B_{R}\right)\right)}+\left\|f_{0}\right\|_{L^{1, n /(n+2)}\left(\Omega_{T}\right)}\right)
$$

for any $0<\rho \leqslant R \leqslant \min \left(R_{\Omega}, \sqrt{t_{0}}\right)$.

Proposition 11. For $x_{0} \in \partial \Omega, t_{0}=0$ and $Q_{R}=B_{R}\left(x_{0}\right) \cap \Omega \times\left[0, R^{2}\right]$, we have

$$
\frac{1}{\rho^{n}}\|u\|_{L^{\infty}\left(\left(0, \rho^{2}\right) ; L^{1}\left(B_{\rho}\right)\right)} \leqslant C\left(\frac{1}{R^{n}}\|u\|_{L^{\infty}\left(\left(0, R^{2}\right) ; L^{1}\left(B_{R}\right)\right)}+\left\|f_{0}\right\|_{L^{1, n /(n+2)}\left(\Omega_{T}\right)}\right)
$$

for any $0<\rho \leqslant R \leqslant \min \left(R_{\Omega}, \sqrt{T}\right)$.

4.2. BMO estimates via $\mathcal{L}^{1,1}$. We combine the local $L^{1}$ estimates obtained in the last subsection to derive a global BMO estimate of $u$, uniform with respect to time.

Proposition 12. Propositions 8-11 imply that the solution of (10) satisfies

$$
\|u\|_{L^{\infty}((0, T) ; \overline{\mathrm{BMO}})} \leqslant C\left\|f_{0}\right\|_{L^{1, n /(n+2)\left(\Omega_{T}\right)}},
$$

where $C$ depends only on $K, n$ and $\Omega$ (independent of $T$ ).

Proof. Set $M=\left\|f_{0}\right\|_{L^{1, n /(n+2)(}\left(\Omega_{T}\right)}$.

First, we prove the proposition for $T \geqslant R_{\Omega}^{2}$. We shall prove that for $R<R_{\Omega} / 2$ and any set $B_{R}=B_{R}\left(x_{0}\right) \cap \Omega$ with some point $x_{0} \in \bar{\Omega}$ and $\delta=\operatorname{dist}\left(x_{0}, \partial \Omega\right)$, the following estimates hold:

$$
\begin{cases}\frac{1}{R^{n}}\|u\|_{L^{\infty}\left((0, T) ; L^{1}\left(B_{R}\right)\right)} \leqslant C\left(\|u\|_{L^{\infty}\left((0, T) ; L^{1}(\Omega)\right)}+M\right) & \text { if } \delta \leqslant R, \\ \frac{1}{R^{n}}\left\|u-u_{B_{R}}\right\|_{L^{\infty}\left((0, T) ; L^{1}\left(B_{R}\right)\right)} \leqslant C\left(\|u\|_{L^{\infty}\left((0, T) ; L^{1}(\Omega)\right)}+M\right) & \text { if } \delta>R .\end{cases}
$$

Case 1: $\delta \leqslant R$. In this case, there exists a region $B_{2 R}=B_{2 R}\left(y_{0}\right) \cap \Omega$ with some $y_{0} \in \partial \Omega$ such that $B_{R} \subset B_{2 R}$ and so, for any given $t_{0} \in[0, T]$,

$$
\left\|u\left(\cdot, t_{0}\right)\right\|_{L^{1}\left(B_{R}\right)} \leqslant\left\|u\left(\cdot, t_{0}\right)\right\|_{L^{1}\left(B_{2 R}\right)} .
$$


Now if $t_{0} \leqslant 4 R^{2}$, then by Proposition 11 ,

$$
\frac{1}{R^{n}}\left\|u\left(\cdot, t_{0}\right)\right\|_{L^{1}\left(B_{2 R}\right)} \leqslant\|u\|_{L^{\infty}\left(\left(0,4 R^{2}\right) ; L^{1}\left(B_{2 R}\right)\right)} \leqslant C\left(\frac{1}{R_{\Omega}^{n}}\|u\|_{L^{\infty}\left(\left(0, R_{\Omega}^{2}\right) ; L^{1}\left(B_{R_{\Omega}}\right)\right)}+M\right) .
$$

Otherwise, $t_{0}>4 R^{2}$ and by Proposition 10 , for $R_{0}=\min \left(\sqrt{t_{0}}, R_{\Omega}\right)$ and $R_{m}=$ $\max \left(\sqrt{t_{0}}, R_{\Omega}\right)$ we have

$$
\begin{aligned}
& \frac{1}{R^{n}}\|u\|_{L^{\infty}\left(\left(t_{0}-4 R^{2}, t_{0}\right) ; L^{1}\left(B_{2 R}\right)\right)} \\
& \quad \leqslant C\left(\frac{1}{R_{0}^{n}}\|u\|_{\left.L^{\infty}\left(t_{0}-R_{0}^{2}, t_{0}\right) ; L^{1}\left(B_{R_{0}}\right)\right)}+M\right) \\
& \quad= \begin{cases}C\left(\frac{1}{t_{0}^{n / 2}}\|u\|_{L^{\infty}\left(\left(0, t_{0}\right) ; L^{1}\left(B_{\left.\left.\sqrt{t_{0}}\right)\right)}\right.\right.}+M\right), & \sqrt{t_{0}}<R_{\Omega}, \\
C\left(\frac{1}{R_{\Omega}^{n}}\|u\|_{\left.L^{\infty}\left(t_{0}-R_{\Omega}^{2}, t_{0}\right) ; L^{1}\left(B_{R_{\Omega}}\right)\right)}+M\right), & \sqrt{t_{0}} \geqslant R_{\Omega},\end{cases} \\
& \quad \leqslant \begin{cases}C\left(\frac{1}{R_{\Omega}^{n}}\|u\|_{L^{\infty}\left(\left(0, R_{\Omega}\right) ; L^{1}\left(B_{R_{\Omega}}\right)\right)}+M\right), & \sqrt{t_{0}}<R_{\Omega} \\
C\left(\frac{1}{R_{\Omega}^{n}}\|u\|_{L^{\infty}\left(\left(t_{0}-R_{\Omega}^{2}, t_{0}\right) ; L^{1}\left(B_{R_{\Omega}}\right)\right)}+M\right), & \sqrt{t_{0}} \geqslant R_{\Omega} .\end{cases}
\end{aligned}
$$

To conclude, for $\delta \leqslant R$ and $t_{0} \in[0, T]$ we have

$$
\frac{1}{R^{n}}\left\|u\left(\cdot, t_{0}\right)\right\|_{L^{1}\left(B_{R}\right)} \leqslant \frac{C}{R_{\Omega}^{n}}\|u\|_{L^{\infty}\left((0, T) ; L^{1}(\Omega)\right)}+C M .
$$

Case 2: $\delta>R$. In this case, we set $R_{0}=\min \left(\delta, \sqrt{t_{0}}, R_{\Omega}\right)$. Then Proposition 8 implies that

$$
\begin{aligned}
& \frac{1}{R^{n}}\left\|u-u_{B_{R}}\right\|_{L^{\infty}\left(\left(t_{0}-R^{2}, t_{0}\right) ; L^{1}\left(B_{R}\right)\right)} \\
& \quad \leqslant \frac{1}{R^{n}}\left\|u-u_{Q_{R}}\right\|_{L^{\infty}\left(\left(t_{0}-R^{2}, t_{0}\right) ; L^{1}\left(B_{R}\right)\right)} \leqslant C\left(\frac{1}{R_{0}^{n}}\|u\|_{\left.L^{\infty}\left(t_{0}-R_{0}^{2}, t_{0}\right) ; L^{1}\left(B_{R_{0}}\right)\right)}+M\right) \\
& = \begin{cases}C\left(\frac{1}{R_{\Omega}^{n}}\|u\|_{L^{\infty}\left(\left(t_{0}-R_{\Omega}^{2}, t_{0}\right) ; L^{1}\left(B_{R_{\Omega}}\right)\right)}+M\right) & \text { if } R_{\Omega} \leqslant \min \left(\delta, \sqrt{t_{0}}\right), \\
C\left(\frac{1}{\delta^{n}}\|u\|_{L^{\infty}\left(\left(t_{0}-\delta^{2}, t_{0}\right) ; L^{1}\left(B_{\delta}\right)\right)}+M\right) & \text { else if } \delta \leqslant \min \left(\sqrt{t_{0}}, R_{\Omega}\right), \\
C\left(\frac{1}{R_{0}^{n}}\|u\|_{L^{\infty}\left(\left(0, R_{0}^{2}\right) ; L^{1}\left(B_{R_{0}}\right)\right)}+M\right) & \text { else } t_{0}=R_{0}^{2},\end{cases}
\end{aligned}
$$




$$
\begin{aligned}
& \leqslant\left\{\begin{array}{l}
\frac{C}{R_{\Omega}^{n}}\|u\|_{L^{\infty}\left((0, T) ; L^{1}(\Omega)\right)}+C M \quad \text { if } R_{\Omega} \leqslant \min \left(\delta, \sqrt{t_{0}}\right), \\
\frac{C}{R_{\Omega}^{n}}\|u\|_{L^{\infty}\left((0, T) ; L^{1}(\Omega)\right)}+C M \quad \text { else if } \delta \leqslant \min \left(\sqrt{t_{0}}, R_{\Omega}\right) \quad \text { by }(17), \\
\left\{\left(\frac{1}{\delta^{n}}\|u\|_{L^{\infty}\left(\left(0, \delta^{2}\right) ; L^{1}\left(B_{\delta}\right)\right)}+M\right) \quad \text { else if } \delta \leqslant R_{\Omega}\right. \\
C\left(\frac{1}{R_{\Omega}^{n}}\|u\|_{L^{\infty}\left(\left(0, R_{\Omega}^{2}\right) ; L^{1}\left(B_{R_{\Omega}}\right)\right)}+M\right) \text { else if } \delta>R_{\Omega}
\end{array} \quad\right. \text { (by Proposition 11), } \\
& \leqslant
\end{aligned}
$$

So far we have proved (14). Once we note that $\|u\|_{L^{\infty}\left((0, T) ; L^{1}(\Omega)\right)} \leqslant C\left\|f_{0}\right\|_{L^{1}\left(\Omega_{T}\right)}$, we derive (13) from (14).

Second, we prove the proposition for $0<T<R_{\Omega}$. In this case, we consider the solution $\hat{u}$ of the equation

$$
\frac{\partial \hat{u}}{\partial t}-\nabla \cdot(A \nabla \hat{u})=\hat{f}_{0}
$$

in the domain $\Omega_{R_{\Omega}}=\Omega \times\left(0, R_{\Omega}\right)$ with the boundary and initial conditions $\hat{u}=0$ on $\partial \Omega \times\left(0, R_{\Omega}\right)$ and $\hat{u}(x, 0)=0$ for $x \in \Omega$, where

$$
\hat{f}_{0}(x, t)= \begin{cases}f_{0}(x, t) & \text { for } t \in(0, T), \\ 0 & \text { for } t \in\left(T, R_{\Omega}\right) .\end{cases}
$$

Check that

$$
\begin{aligned}
& \left\|\hat{f}_{0}\right\|_{L^{1, n /(n+2)}\left(\Omega_{R_{\Omega}}\right)} \leqslant C\left\|f_{0}\right\|_{L^{1, n /(n+2)}\left(\Omega_{T}\right)}, \\
& \left\|\hat{f}_{0}\right\|_{L^{1}\left(\Omega_{R_{\Omega}}\right)} \leqslant C\left\|f_{0}\right\|_{L^{1}\left(\Omega_{T}\right)}, \\
& \|u\|_{L^{\infty}((0, T) ; \overline{\mathrm{BMO}})} \leqslant\|\hat{u}\|_{L^{\infty}\left(\left(0, R_{\Omega}\right) ; \overline{\mathrm{BMO}}\right)},
\end{aligned}
$$

where the constant $C$ does not depend on $T$ (as $T \rightarrow 0$ ). Then we apply the inequality (13) to $\hat{u}$ with $T=R_{\Omega}$.

4.3. BMO estimates via $\mathcal{L}^{2,1}$. In this section, we present estimates for the solution of (11). The idea is similar to that in Section 4.2. From the proof of the following lemma we can see the main difference between the current subsection and the last subsection.

Lemma 13. Let $x_{0} \in \Omega$ and $0<t_{0}<T$. There exist $\alpha_{0} \in(0,1)$ and $C>0$ such that if $u$ is the solution to (11) in $Q_{R}=B_{R}\left(x_{0}\right) \times I_{R}$ with $I_{R}=\left(t_{0}-R^{2}, t_{0}\right]$, 
then

$$
\max _{t \in I_{\rho}}\left\|u-u_{Q_{\rho}}\right\|_{L^{2}\left(B_{\rho}\right)}^{2} \leqslant C\left(\frac{\rho}{R}\right)^{n+2 \alpha_{0}} \max _{t \in I_{R}}\|u-\theta\|_{L^{2}\left(B_{R}\right)}^{2}+C\|\vec{f}\|_{L^{2}\left(Q_{R}\right)}^{2}
$$

holds for any $0<\rho \leqslant R \leqslant \min \left(\operatorname{dist}\left(x_{0}, \partial \Omega\right), \sqrt{t_{0}}\right)$, where $C$ depends only on $K$ and $n$.

Proof. Let $\widetilde{B}_{r}=B_{r}(0), \widetilde{I}_{r}=\left(-r^{2}, 0\right]$ and $\widetilde{\Gamma}_{r}=\partial \widetilde{B}_{r} \times \widetilde{I}_{r}$. With any function $w$ defined on $Q_{R}$, we associate a function $\tilde{\xi}(y, s)=\xi\left(x_{0}+R y, t_{0}+R^{2} s\right)$ defined on $\widetilde{Q}_{1}:=\widetilde{B}_{1} \times \widetilde{I}_{1}$. Then $\tilde{u}$ is a solution of the equation

$$
\frac{\partial \tilde{u}}{\partial s}-\nabla_{y} \cdot\left(\tilde{A} \nabla_{y} \tilde{u}\right)=R \nabla_{y} \cdot \tilde{f}
$$

in $\widetilde{Q}_{1}$. Let $w$ be the solution of

$$
\frac{\partial w}{\partial s}-\nabla_{y} \cdot\left(\tilde{A} \nabla_{y} w\right)=R \nabla_{y} \cdot \tilde{f}
$$

with the initial and boundary condition $w=0$ on the parabolic boundary $\partial_{\mathrm{p}} \widetilde{Q}_{1}$. Multiplying the above equation by $w$ and integrating the result over $\widetilde{Q}_{1}$, we obtain

$$
\|w\|_{L^{\infty}\left(\widetilde{I}_{1} ; L^{2}\left(\widetilde{B}_{1}\right)\right)} \leqslant C R\|\tilde{f}\|_{L^{2}\left(\widetilde{Q}_{1}\right)} .
$$

On the other hand, we observe that $v=\tilde{u}-\tilde{u}_{\widetilde{Q}_{1}}-w$ is the solution of

$$
\frac{\partial v}{\partial s}-\nabla_{y} \cdot\left(\tilde{A} \nabla_{y} v\right)=0
$$

in $\widetilde{Q}_{1}$. By the De Giorgi-Nash estimates of parabolic equations, we know that there exists $\alpha_{0} \in(0,1)$ such that for $\rho \in(0,1 / 2]$,

$$
\begin{aligned}
\max _{t \in I_{\rho}} \frac{1}{\rho^{n+2 \alpha_{0}}} \int_{\widetilde{B}_{\rho}}\left|v-v_{\widetilde{Q}_{\rho}}\right|^{2} d y & \leqslant C|v|_{C^{\alpha_{0}, \alpha_{0} / 2}\left(\widetilde{Q}_{1 / 2}\right)}^{2} \\
& \leqslant C\|v\|_{L^{2}\left(\widetilde{Q}_{1}\right)}^{2} \leqslant C \max _{t \in \widetilde{I}_{1}}\|v\|_{L^{2}\left(\widetilde{B}_{1}\right)}^{2} .
\end{aligned}
$$

Therefore,

$$
\begin{aligned}
\max _{t \in \widetilde{I}_{\rho}}\left\|\tilde{u}-\tilde{u}_{\widetilde{Q}_{\rho}}\right\|_{L^{2}\left(\widetilde{B}_{\rho}\right)}^{2} & \leqslant C \max _{t \in \widetilde{I}_{\rho}}\left\|v-v \widetilde{Q}_{\widetilde{Q}_{\rho}}\right\|_{L^{2}\left(\widetilde{B}_{\rho}\right)}^{2}+C \max _{t \in \widetilde{I}_{\rho}}\left\|w-w_{\widetilde{Q}_{\rho}}\right\|_{L^{2}\left(\widetilde{B}_{\rho}\right)}^{2} \\
& \leqslant C \rho^{n+2 \alpha_{0}} \max _{t \in \widetilde{I}_{1}}\|v\|_{L^{2}\left(\widetilde{B}_{1}\right)}^{2}+C \max _{t \in \widetilde{I}_{1}}\|w\|_{L^{2}\left(\widetilde{B}_{1}\right)}^{2}
\end{aligned}
$$




$$
\begin{aligned}
& \leqslant C \rho^{n+2 \alpha_{0}} \max _{t \in \widetilde{I}_{1}}\left\|\tilde{u}-\tilde{u} \widetilde{Q}_{1}\right\|_{L^{2}\left(\widetilde{B}_{1}\right)}^{2}+C \max _{t \in \widetilde{I}_{1}}\|w\|_{L^{2}\left(\widetilde{B}_{1}\right)}^{2} \\
& \leqslant C \rho^{n+2 \alpha_{0}} \max _{t \in \widetilde{I}_{1}}\left\|\tilde{u}-\tilde{u} \widetilde{Q}_{1}\right\|_{L^{2}\left(\widetilde{B}_{1}\right)}^{2}+C R^{2}\|\tilde{f}\|_{L^{2}\left(\widetilde{Q}_{1}\right)}^{2} \\
& \leqslant C \rho^{n+2 \alpha_{0}} \max _{t \in \widetilde{I}_{1}}\|\tilde{u}\|_{L^{2}\left(\widetilde{B}_{1}\right)}^{2}+C R^{2}\|\tilde{f}\|_{L^{2}\left(\widetilde{Q}_{1}\right)}^{2} .
\end{aligned}
$$

Transforming back to the $(x, t)$-coordinates, we complete the proof of the lemma for $\theta=0$. Then we note that $u-\theta$ is also a solution to Equation (11) in $Q_{R}$ for any $\theta \in \mathbb{R}$.

In a similar way, we can prove the following lemmas and propositions.

LEMMA 14. Let $x_{0} \in \Omega$ and $t_{0}=0$. There exist $\alpha_{0} \in(0,1)$ and $C>0$ such that if $u$ is the solution of (11) in $Q_{R}=B_{R}\left(x_{0}\right) \times \underline{I}_{R}$ with $\underline{I}_{R}=\left[0, R^{2}\right]$, then

$$
\max _{t \in \underline{I}_{\rho}}\|u\|_{L^{2}\left(B_{\rho}\right)}^{2} \leqslant C\left(\frac{\rho}{R}\right)^{n+2 \alpha_{0}} \max _{t \in \underline{I}_{R}}\|u\|_{L^{2}\left(B_{R}\right)}^{2}+C\|\vec{f}\|_{L^{2}\left(Q_{R}\right)}^{2}
$$

holds for any $0<\rho \leqslant R \leqslant \min \left(\operatorname{dist}\left(x_{0}, \partial \Omega\right), \sqrt{T}\right)$, where $C$ and $\alpha_{0}$ depend only on $K$ and $n$.

LEMMA 15. Let $x_{0} \in \partial \Omega$ and $t_{0}>0$. There exist $\alpha_{0} \in(0,1)$ and $C>0$ such that if $u$ is the solution of (11) in $Q_{R}=B_{R} \times I_{R}$ with $B_{R}=B_{R}\left(x_{0}\right) \cap \Omega$ and $I_{R}=\left(t_{0}-R^{2}, t_{0}\right]$, then

$$
\max _{t \in I_{\rho}}\|u\|_{L^{2}\left(B_{\rho}\right)}^{2} \leqslant C\left(\frac{\rho}{R}\right)^{n+2 \alpha_{0}} \max _{t \in I_{R}}\|u\|_{L^{2}\left(B_{R}\right)}^{2}+C\|\vec{f}\|_{L^{2}\left(Q_{R}\right)}^{2}
$$

holds for any $0<\rho \leqslant R \leqslant \min \left(R_{\Omega}, \sqrt{t_{0}}\right)$, where $C$ and $\alpha_{0}$ depend only on $K, n$ and $\Omega$.

LEMMA 16. Let $x_{0} \in \partial \Omega$ and $t_{0}=0$. There exist $\alpha_{0} \in(0,1)$ and $C>0$ such that if $u$ is the solution to (11) in $Q_{R}=B_{R} \times \underline{I}_{R}$, with $B_{R}=B_{R}\left(x_{0}\right) \cap \Omega$ and $\underline{I}_{R}=\left[0, R^{2}\right]$, then

$$
\max _{t \in \underline{I}_{\rho}}\|u\|_{L^{2}\left(B_{\rho}\right)}^{2} \leqslant C\left(\frac{\rho}{R}\right)^{n+2 \alpha_{0}} \max _{t \in \underline{I}_{R}}\|u\|_{L^{2}\left(B_{R}\right)}^{2}+C\|\vec{f}\|_{L^{2}\left(Q_{R}\right)}^{2}
$$

holds for any $0<\rho \leqslant R \leqslant \min \left(R_{\Omega}, \sqrt{T}\right)$, where $C$ and $\alpha_{0}$ depend only on $K, n$ and $\Omega$.

From the above lemmas, using Lemma 7 we can derive the following results concerning the solution of (11). 
Proposition 17. For $x_{0} \in \Omega, t_{0}>0, Q_{R}=B_{R}\left(x_{0}\right) \times\left(t_{0}-R^{2}, t_{0}\right]$ and $0<\rho \leqslant$ $R \leqslant \min \left(\operatorname{dist}\left(x_{0}, \partial \Omega\right), \sqrt{t_{0}}\right)$, we have

$$
\begin{aligned}
\| u & -u_{Q_{\rho}} \|_{L^{\infty}\left(\left(t_{0}-\rho^{2}, t_{0}\right) ; L^{2}\left(B_{\rho}\right)\right)}^{2} \\
& \leqslant C\left(\frac{1}{R^{n}}\|u\|_{\left.L^{\infty}\left(t_{0}-R^{2}, t_{0}\right) ; L^{2}\left(B_{R}\right)\right)}^{2}+\|\vec{f}\|_{L^{2, n /(n+2)}\left(\Omega_{T}\right)}^{2}\right) \rho^{n} .
\end{aligned}
$$

Proposition 18. For $x_{0} \in \Omega, t_{0}=0, Q_{R}=B_{R}\left(x_{0}\right) \times\left[0, R^{2}\right]$ and $0<\rho \leqslant R \leqslant$ $\min \left(\operatorname{dist}\left(x_{0}, \partial \Omega\right), \sqrt{T}\right)$, we have

$$
\|u\|_{L^{\infty}\left(\left(0, \rho^{2}\right) ; L^{2}\left(B_{\rho}\right)\right)}^{2} \leqslant C\left(\frac{1}{R^{n}}\|u\|_{L^{\infty}\left(\left(0, R^{2}\right) ; L^{2}\left(B_{R}\right)\right)}^{2}+\|\vec{f}\|_{L^{2, n /(n+2)}\left(\Omega_{T}\right)}^{2}\right) \rho^{n} .
$$

Proposition 19. For $x_{0} \in \partial \Omega, t_{0}>0, Q_{R}=B_{R}\left(x_{0}\right) \cap \Omega \times\left(t_{0}-R^{2}, t_{0}\right]$ and $0<\rho<R \leqslant \min \left(R_{\Omega}, \sqrt{t_{0}}\right)$, we have

$$
\|u\|_{L^{\infty}\left(\left(t_{0}-\rho^{2}, t_{0}\right) ; L^{2}\left(B_{\rho}\right)\right)}^{2} \leqslant C\left(\frac{1}{R^{n}}\|u\|_{L^{\infty}\left(\left(t_{0}-R^{2}, t_{0}\right) ; L^{2}\left(B_{R}\right)\right)}^{2}+\|\vec{f}\|_{L^{2, n /(n+2)}\left(\Omega_{T}\right)}^{2}\right) \rho^{n} .
$$

Proposition 20. For $x_{0} \in \partial \Omega, t_{0}=0, Q_{R}=B_{R}\left(x_{0}\right) \cap \Omega \times\left[0, R^{2}\right]$ and $0<\rho<R \leqslant \min \left(R_{\Omega}, \sqrt{T}\right)$, we have

$$
\|u\|_{L^{\infty}\left(\left(0, \rho^{2}\right) ; L^{2}\left(B_{\rho}\right)\right)}^{2} \leqslant C\left(\frac{1}{R^{n}}\|u\|_{L^{\infty}\left(\left(0, R^{2}\right) ; L^{2}\left(B_{R}\right)\right)}^{2}+\|\vec{f}\|_{L^{2, n /(n+2)}\left(\Omega_{T}\right)}^{2}\right) \rho^{n} .
$$

With the above propositions and following the outline of Section 4.2, we can prove the global BMO estimate below.

Proposition 21. Propositions 17-20 imply that the solution of (11) satisfies

$$
\|u\|_{L^{\infty}((0, T) ; \overline{\mathrm{BMO}})} \leqslant C\|\vec{f}\|_{L^{2, n /(n+2)\left(\Omega_{T}\right)}},
$$

where $C$ depends only on $K, n$ and $\Omega$ (independent of $T$ ).

\section{Hölder estimate of parabolic equations}

In this section, we list the propositions to be used in deriving (7). We omit the proof of these propositions, as it is very similar to the proof presented in the last section. The reason we keep these propositions in this section is that some of them are also used in the next section to prove global well-posedness of the degenerate thermistor problem.

There exist positive constants $\alpha_{0}$ and $C$ such that the following propositions hold. 
Proposition 22. For $x_{0} \in \Omega, t_{0}>0, Q_{R}=B_{R}\left(x_{0}\right) \times\left(t_{0}-R^{2}, t_{0}\right], 0<2 \rho \leqslant$ $R \leqslant \min \left(\operatorname{dist}\left(x_{0}, \partial \Omega\right), \sqrt{t_{0}}\right)$ and $0<\alpha<\alpha_{0}$, the solution of (10) satisfies

$$
\frac{1}{\rho^{n+2+\alpha}}\left\|u-u_{Q_{\rho}}\right\|_{L^{1}\left(Q_{\rho}\right)} \leqslant C\left(\frac{1}{R^{n+2+\alpha}}\|u-\theta\|_{L^{1}\left(Q_{R}\right)}+\frac{1}{R^{n+\alpha}}\left\|f_{0}\right\|_{L^{1}\left(Q_{R}\right)}\right),
$$

where $\theta$ is an arbitrary constant.

Proposition 23. For $x_{0} \in \Omega, t_{0}=0, Q_{R}=B_{R}\left(x_{0}\right) \times\left[0, R^{2}\right], 0<\rho \leqslant R \leqslant$ $\min \left(\operatorname{dist}\left(x_{0}, \partial \Omega\right), \sqrt{T}\right)$ and $0<\alpha<\alpha_{0}$, the solution of (10) satisfies

$$
\frac{1}{\rho^{n+2+\alpha}}\|u\|_{L^{1}\left(Q_{\rho}\right)} \leqslant C\left(\frac{1}{R^{n+2+\alpha}}\|u\|_{L^{1}\left(Q_{R}\right)}+\frac{1}{R^{n+\alpha}}\left\|f_{0}\right\|_{L^{1}\left(Q_{R}\right)}\right) .
$$

Proposition 24. For $x_{0} \in \partial \Omega, t_{0}>0, Q_{R}=B_{R}\left(x_{0}\right) \cap \Omega \times\left(t_{0}-R^{2}, t_{0}\right]$, $0<\rho \leqslant R \leqslant \min \left(R_{\Omega}, \sqrt{t_{0}}\right)$ and $0<\alpha<\alpha_{0}$, the solution of (10) satisfies

$$
\frac{1}{\rho^{n+2+\alpha}}\|u\|_{L^{1}\left(Q_{\rho}\right)} \leqslant C\left(\frac{1}{R^{n+2+\alpha}}\|u\|_{L^{1}\left(Q_{R}\right)}+\frac{1}{R^{n+\alpha}}\left\|f_{0}\right\|_{L^{1}\left(Q_{R}\right)}\right) .
$$

Proposition 25. For $x_{0} \in \partial \Omega, t_{0}=0, Q_{R}=B_{R}\left(x_{0}\right) \cap \Omega \times\left[0, R^{2}\right], 0<\rho \leqslant$ $R \leqslant \min \left(R_{\Omega}, \sqrt{T}\right)$ and $0<\alpha<\alpha_{0}$, the solution of (10) satisfies

$$
\frac{1}{\rho^{n+2+\alpha}}\|u\|_{L^{1}\left(Q_{\rho}\right)} \leqslant C\left(\frac{1}{R^{n+2+\alpha}}\|u\|_{L^{1}\left(Q_{R}\right)}+\frac{1}{R^{n+\alpha}}\left\|f_{0}\right\|_{L^{1}\left(Q_{R}\right)}\right) .
$$

With the above propositions and following the outline of Section 4.2, we can derive the following estimate in terms of the Campanato space.

PROPOSITION 26. The solution of (10) satisfies

$$
\|u\|_{\mathcal{L}_{\text {para }}^{1,1+\alpha /(n+2)}\left(\Omega_{T}\right)} \leqslant C\left\|f_{0}\right\|_{L^{1,(n+\alpha) /(n+2)\left(\Omega_{T}\right)}},
$$

where $C$ depends only on $K, n$ and $\Omega$ (independent of $T$ ).

The local and global estimates in $\mathcal{L}_{\text {para }}^{2, \theta}\left(\Omega_{T}\right)$ follow in a similar way. To conclude, we have the following.

Proposition 27. For $x_{0} \in \Omega, t_{0}>0, Q_{R}=B_{R}\left(x_{0}\right) \times\left(t_{0}-R^{2}, t_{0}\right], 0<2 \rho \leqslant$ $R \leqslant \min \left(\operatorname{dist}\left(x_{0}, \partial \Omega\right), \sqrt{t_{0}}\right)$ and $0<\alpha<\alpha_{0}$, the solution of (11) satisfies

$$
\frac{1}{\rho^{n+2+2 \alpha}}\left\|u-u_{Q_{\rho}}\right\|_{L^{2}\left(Q_{\rho}\right)}^{2} \leqslant C\left(\frac{1}{R^{n+2+2 \alpha}}\|u-\theta\|_{L^{2}\left(Q_{R}\right)}^{2}+\frac{1}{R^{n+2 \alpha}}\|\vec{f}\|_{L^{2}\left(Q_{R}\right)}^{2}\right),
$$

where $\theta$ is an arbitrary constant. 
Proposition 28. For $x_{0} \in \Omega, t_{0}=0, Q_{R}=B_{R}\left(x_{0}\right) \cap \Omega \times\left[0, R^{2}\right], 0<\rho \leqslant$ $R \leqslant \min \left(\operatorname{dist}\left(x_{0}, \partial \Omega\right), \sqrt{T}\right)$ and $0<\alpha<\alpha_{0}$, the solution of (11) satisfies

$$
\frac{1}{\rho^{n+2+2 \alpha}}\|u\|_{L^{2}\left(Q_{\rho}\right)}^{2} \leqslant C\left(\frac{1}{R^{n+2+2 \alpha}}\|u\|_{L^{2}\left(Q_{R}\right)}^{2}+\frac{1}{R^{n+2 \alpha}}\|\vec{f}\|_{L^{2}\left(Q_{R}\right)}^{2}\right) .
$$

Proposition 29. For $x_{0} \in \partial \Omega, t_{0}>0, Q_{R}=B_{R}\left(x_{0}\right) \cap \Omega \times\left(t_{0}-R^{2}, t_{0}\right]$, $0<\rho<R \leqslant \min \left(R_{\Omega}, \sqrt{t_{0}}\right)$ and $0<\alpha<\alpha_{0}$, the solution of (11) satisfies

$$
\frac{1}{\rho^{n+2+2 \alpha}}\|u\|_{L^{1}\left(Q_{\rho}\right)} \leqslant C\left(\frac{1}{R^{n+2+2 \alpha}}\|u\|_{L^{2}\left(Q_{R}\right)}^{2}+\frac{1}{R^{n+2 \alpha}}\|\vec{f}\|_{L^{2}\left(Q_{R}\right)}^{2}\right) .
$$

Proposition 30. For $x_{0} \in \partial \Omega, t_{0}=0, Q_{R}=B_{R}\left(x_{0}\right) \cap \Omega \times\left[0, R^{2}\right], 0<\rho \leqslant$ $R \leqslant \min \left(R_{\Omega}, \sqrt{T}\right)$ and $0<\alpha<\alpha_{0}$, the solution of (11) satisfies

$$
\frac{1}{\rho^{n+2+2 \alpha}}\|u\|_{L^{2}\left(Q_{\rho}\right)}^{2} \leqslant C\left(\frac{1}{R^{n+2+2 \alpha}}\|u\|_{L^{2}\left(Q_{R}\right)}^{2}+\frac{1}{R^{n+2 \alpha}}\|\vec{f}\|_{L^{2}\left(Q_{R}\right)}^{2}\right) .
$$

PROPOSITION 31. The solution of (11) satisfies

$$
\|u\|_{\mathcal{L}_{\text {para }}^{2,1+2 \alpha /(n+2)}\left(\Omega_{T}\right)} \leqslant C\|\vec{f}\|_{L^{2,(n+2 \alpha) /(n+2)\left(\Omega_{T}\right)}},
$$

where $C$ depends only on $K, n$ and $\Omega$ (independent of $T$ ).

Propositions 26 and 31 imply the global Hölder estimate (7).

\section{The degenerate thermistor problem}

In this section, we prove Theorem 2 concerning global well-posedness of the degenerate thermistor problem. Before we prove the theorem, we introduce some lemmas to be used.

\subsection{Preliminaries}

LEMmA 32. Let $p>0$. If $u \in \operatorname{BMO}\left(\mathbb{R}^{n}\right), u \geqslant 0$, and $C_{1}+C_{2}|s|^{p} \leqslant \rho(s) \leqslant$ $C_{3}+C_{4}|s|^{p}$ for $s \geqslant 0$, then $\rho(u)$ is an $A_{2}$ weight in the sense that

$$
\left(\frac{1}{|B|} \int_{B} \rho(u) d x\right)\left(\frac{1}{|B|} \int_{B} \frac{1}{\rho(u)} d x\right) \leqslant C
$$

for any ball $B \subset \mathbb{R}^{n}$, where the constant $C$ depends on $C_{1}, C_{2}, C_{3}, C_{4}, p$ and $\|u\|_{\text {ВMO. }}$ 
Proof. For any ball $B \subset \mathbb{R}^{n}$, we set $B_{1}=\left\{x \in B|| u(x)-u_{B} \mid<\frac{1}{2} u_{B}\right\}$ and $B_{2}=B \backslash B_{1}$. By the Nirenberg inequality [13] we have $\left|B_{2}\right| /|B| \leqslant e^{-C u_{B} /\|u\|_{\text {вмо }}}$. Clearly, $\rho(u) \geqslant C \rho\left(u_{B}\right)$ on $B_{1}$. Therefore,

$$
\begin{aligned}
\frac{1}{|B|} \int_{B} \rho(u) d x & \leqslant \frac{C}{|B|} \int_{B}\left(1+\left|u-u_{B}\right|^{p}\right) d x+\frac{C}{|B|} \int_{B}\left|u_{B}\right|^{p} d x \\
& \leqslant C+C\left|u_{B}\right|^{p} \leqslant C \rho\left(u_{B}\right), \\
\frac{1}{|B|} \int_{B} \frac{1}{\rho(u)} d x & \leqslant \frac{1}{|B|} \int_{B_{1}} \frac{1}{\rho(u)} d x+\frac{C\left|B_{2}\right|}{|B|} \\
& \leqslant \frac{C}{\rho\left(u_{B}\right)}+e^{-C u_{B} /\|u\|_{\text {вмо }}} \leqslant \frac{C}{\rho\left(u_{B}\right)} .
\end{aligned}
$$

The last two inequalities imply that $\rho(u)$ is an $A_{2}$ weight.

The following lemma concerns maximal regularity of parabolic equations, which is an application of the maximal regularity of [26] and [17] (with the perturbation method for the treatment of operators with merely continuous coefficients).

LEMMA 33. Let $u$ be the solution of the parabolic problem (4) in $\mathbb{R}^{n}(n=2,3)$ with the Dirichlet boundary/initial conditions $u \equiv g \equiv f_{0} \equiv 0$, and assume that the coefficient matrix A is continuous. Then we have

$$
\|u\|_{L^{p}\left(I ; W^{1, q}(\Omega)\right)} \leqslant C_{p, q}\|\vec{f}\|_{L^{p}\left(I ; L^{q}(\Omega)\right)}
$$

for some $q>n$ and any $1<p<\infty$. The constant $C_{p, q}$ depends only on $p, q, K$, the domain $\Omega$ and the modulo of continuity of $A$.

The analogous result for elliptic equations is given below, which can be proved by applying the $W^{1, q}$ estimate of [17] with a perturbation argument.

Lemma 34. Let $A_{i j}, i, j=1, \ldots, n$, be continuous functions defined on $\Omega$, satisfying

$$
K^{-1}|\xi|^{2} \leqslant \sum_{i, j=1}^{n} A_{i j}(x) \xi_{i} \xi_{j} \leqslant K|\xi|^{2} \quad \text { for all } \xi \in \mathbb{R}^{n} \text {, a.e. } x \in \mathbb{R}^{n}(n=2,3),
$$

where $K$ is a positive constant. Let $u$ be the solution of the elliptic equation

$$
-\nabla \cdot(A \nabla u)=\nabla \cdot \vec{f} \quad \text { in } \Omega,
$$

with the Dirichlet boundary condition $u=0$ on $\partial \Omega$. Then we have

$$
\|u\|_{W^{1, q}(\Omega)} \leqslant C_{q}\|\vec{f}\|_{L^{q}(\Omega)}
$$


for some $q>n$. The constant $C_{q}$ depends only on $q, \Lambda$, the domain $\Omega$ and the modulo of continuity of $A$.

The following lemma is concerned with Hölder estimates for inhomogeneous parabolic equations [6], which is also a consequence of Theorem 1.

LEMMA 35. The solution of (4) with $u_{0} \equiv g \equiv 0$ satisfies

$$
\|u\|_{C^{\alpha, \alpha / 2}\left(\bar{\Omega}_{T}\right)} \leqslant C\left(\left\|f_{0}\right\|_{L^{p}\left((0, T) ; L^{q}(\Omega)\right)}+\|\vec{f}\|_{L^{2 p}\left((0, T) ; L^{2 q}(\Omega)\right)}\right),
$$

for some $0<\alpha<1$, provided $1 \leqslant p, q \leqslant \infty$ and $2 / p+n / q<2$.

The following lemma concerns an estimate of $\nabla u$ in the Morrey space for the parabolic equation (4), which was proved in [29] for $u_{0} \equiv g \equiv f_{0} \equiv 0$.

LEMMA 36. The solution of (4) with $f_{0} \equiv 0$ satisfies

$$
\begin{aligned}
\|\nabla u\|_{L_{\mathrm{para}}^{2, n /(n+2)}\left(\Omega_{T}\right)} \leqslant & C\left(\|\vec{f}\|_{L_{\mathrm{para}}^{2, n /(n+2)}\left(\Omega_{T}\right)}+\|\nabla g\|_{L_{\mathrm{para}}^{2, n /(n+2)}\left(\Omega_{T}\right)}\right. \\
& \left.+\left\|\partial_{t} g\right\|_{L_{\mathrm{para}}^{2, n /(n+2)}\left(\Omega_{T}\right)}+\left\|u_{0}\right\|_{L^{\infty}(\Omega)}\right) .
\end{aligned}
$$

6.2. Construction of approximating solutions. For the nondegenerate problem, the existence of a $C^{\alpha}$ solution was proved by Yuan and Lin [30, 31]. Based on their result, for any given $\varepsilon>0$, there exists a weak solution $\left(u^{\varepsilon}, \phi^{\varepsilon}\right)$ such that $\phi^{\varepsilon} \in L^{\infty}\left((0, T) ; H^{1}(\Omega)\right)$ and $u^{\varepsilon} \in C^{\alpha, \alpha / 2}\left(\bar{\Omega}_{T}\right) \cap L^{2}\left((0, T) ; H^{1}(\Omega)\right)$, to the following equations

$$
\begin{aligned}
& \begin{cases}\frac{\partial u^{\varepsilon}}{\partial t}-\nabla \cdot\left(\kappa\left(u^{\varepsilon}\right) \nabla u^{\varepsilon}\right)=\nabla \cdot\left[\left(\sigma\left(u^{\varepsilon}\right)+\varepsilon\right) \phi^{\varepsilon} \nabla \phi^{\varepsilon}\right] & \text { in } \Omega, \\
u^{\varepsilon}=g & \text { on } \partial \Omega, \\
u^{\varepsilon}(x, 0)=u_{0}(x) & \text { for } x \in \Omega,\end{cases} \\
& \begin{cases}-\nabla \cdot\left(\left(\sigma\left(u^{\varepsilon}\right)+\varepsilon\right) \nabla \phi^{\varepsilon}\right)=0 & \text { in } \Omega, \\
\phi^{\varepsilon}=h & \text { on } \partial \Omega .\end{cases}
\end{aligned}
$$

We also note that, by the maximum principle, the solution $u^{\varepsilon}$ of (22) satisfies

$$
u^{\varepsilon} \geqslant c:=\min \left(\min _{x \in \Omega} u_{0}(x), \min _{x \in \partial \Omega} g(x)\right)>0,
$$

and the solution $\phi$ of (23) satisfies

$$
\left\|\phi^{\varepsilon}\right\|_{L^{\infty}\left(\Omega_{T}\right)} \leqslant\|h\|_{L^{\infty}\left(\Gamma_{T}\right)} .
$$


By hypotheses (H1) and (H2), we have

$$
\kappa_{0} \leqslant \kappa\left(u^{\varepsilon}\right) \leqslant \kappa_{1}, \quad \varepsilon \leqslant \sigma\left(u^{\varepsilon}\right)+\varepsilon \leqslant 2 \sigma_{0}:=\sup _{s \geqslant c} \sigma(s),
$$

for some positive constants $\kappa_{0}, \kappa_{1}$ and $\sigma_{0}$, where we choose $\varepsilon<\sigma_{0}$.

Proposition 37. The solution $\left(u^{\varepsilon}, \phi^{\varepsilon}\right)$ of (22) and (23) satisfies

$$
\begin{aligned}
& \left\|u^{\varepsilon}\right\|_{C^{\alpha, \alpha / 2}\left(\bar{\Omega}_{T}\right)}+\left\|u^{\varepsilon}\right\|_{L^{p}\left((0, T) ; W^{1, q}(\Omega)\right)} \\
& \quad+\left\|\partial_{t} u^{\varepsilon}\right\|_{L^{p}\left((0, T) ; W^{-1, q}(\Omega)\right)}+\left\|\phi^{\varepsilon}\right\|_{L^{\infty}\left((0, T) ; W^{1, q}(\Omega)\right)} \leqslant C,
\end{aligned}
$$

and

$$
\left\|\phi^{\varepsilon}\right\|_{C^{\alpha, \alpha / 2}\left(\bar{B}_{R} \times[0, T]\right)} \leqslant C_{\text {dist }\left(\bar{B}_{R}, \partial \Omega\right)}
$$

for any closed ball $\bar{B}_{R} \subset \Omega$, where the constants $C$ and $C_{\operatorname{dist}\left(\bar{B}_{R}, \partial \Omega\right)}$ are independent of $\varepsilon$.

Proof. First, we show that $\sigma\left(u^{\varepsilon}\right)+\varepsilon$ is an $A_{2}$ weight, uniformly with respect to time and $\varepsilon$.

Let $x_{0} \in \Omega, t_{0}>0$ and let $R_{0}=\frac{1}{2} \min \left(\sqrt{t_{0}}, \operatorname{dist}\left(x_{0}, \partial \Omega\right)\right)$. For any ball $B_{R}$ of radius $R$ centered at $x_{0}$, we let $\zeta$ be a smooth function defined on $\mathbb{R}^{n}$, which satisfies $0 \leqslant \zeta \leqslant 1, \zeta=1$ in $B_{R}$ and $\zeta=0$ outside $B_{2 R}$. For any interval $I_{R}=\left(t_{0}-\right.$ $R^{2}, t_{0}$ ], we let $\chi$ be a smooth function defined on $\mathbb{R}$, which satisfies $0 \leqslant \chi \leqslant 1$, $\chi=1$ on $I_{R}$ and $\chi=0$ on $\left(-\infty, t_{0}-4 R^{2}\right]$. Let $Q_{R}=B_{R} \times I_{R}$ so that $\left(u^{\varepsilon}, \phi^{\varepsilon}\right)$ is a solution of (22) and (23) in $Q_{2 R_{0}}$. Multiplying (23) by $\varphi=\phi^{\varepsilon} \zeta^{2}$, we obtain

$$
\int_{B_{R}}\left(\sigma\left(u^{\varepsilon}\right)+\varepsilon\right)\left|\nabla \phi^{\varepsilon}\right|^{2} d x \leqslant \int_{B_{2 R}}\left(\sigma\left(u^{\varepsilon}\right)+\varepsilon\right)\left|\phi^{\varepsilon}\right|^{2}|\nabla \zeta|^{2} d x \leqslant C\left\|\phi^{\varepsilon}\right\|_{L^{\infty}(\Omega)}^{2} R^{n-2} .
$$

Integrating the above inequality with respect to time and using (25), we get

$$
\iint_{Q_{R}}\left(\sigma\left(u^{\varepsilon}\right)+\varepsilon\right)\left|\nabla \phi^{\varepsilon}\right|^{2} d x d t \leqslant C\|h\|_{L^{\infty}\left(\Gamma_{T}\right)}^{2} R^{n} .
$$

Similarly, for $x_{0} \in \partial \Omega, t_{0}>0, R<\frac{1}{2} \min \left(\sqrt{t_{0}}, R_{\Omega}\right), B_{R}:=B_{R}\left(x_{0}\right) \cap \Omega$ and $Q_{R}=Q_{R}\left(x_{0}, t_{0}\right) \cap \Omega_{T}$, we also have (27). From the last inequality we see that

$$
\left\|\sqrt{\sigma\left(u^{\varepsilon}\right)+\varepsilon} \nabla \phi^{\varepsilon}\right\|_{L_{\text {para }}^{2, n /(n+2)}\left(\Omega_{T}\right)} \leqslant C .
$$

By Theorem 1, the solution of (22) satisfies

$$
\begin{aligned}
\left\|u^{\varepsilon}\right\|_{L^{\infty}((0, T) ; \overline{\mathrm{BMO}})} \leqslant & C\left\|\sqrt{\sigma\left(u^{\varepsilon}\right)+\varepsilon} \nabla \phi^{\varepsilon}\right\|_{L_{\mathrm{para}}^{2, n /(n+2)}\left(\Omega_{T}\right)} \\
& +C\left\|u_{0}\right\|_{L^{\infty}(\Omega)}+C\|g\|_{L^{\infty}\left(\Gamma_{T}\right)} \leqslant C .
\end{aligned}
$$


Applying Lemma 36 to Equation (22) and using (28), we derive

$$
\left\|\nabla u^{\varepsilon}\right\|_{L_{\mathrm{para}}^{2, n /(n+2)}\left(\Omega_{T}\right)} \leqslant C\left\|\sqrt{\sigma\left(u^{\varepsilon}\right)+\varepsilon} \nabla \phi^{\varepsilon}\right\|_{L_{\text {para }}^{2, n /(n+2)}\left(\Omega_{T}\right)}+C \leqslant C .
$$

We extend the function $u^{\varepsilon}$ defined on $\Omega$ to $\mathbb{R}^{n}$ by setting $u^{\varepsilon}(x)=c$ for $x \in \mathbb{R}^{n} \backslash \Omega$ so that

$$
\left\|u^{\varepsilon}\right\|_{L^{\infty}\left((0, T) ; \mathrm{BMO}\left(\mathbb{R}^{n}\right)\right)} \leqslant C .
$$

Since (8) holds, from Lemma 32 we see that $\rho\left(u^{\varepsilon}\right)$ (and also $\sigma\left(u^{\varepsilon}\right)=1 / \rho\left(u^{\varepsilon}\right)$ ) is an $A_{2}$ weight uniform with respect to time and $\varepsilon$. It follows that, for any ball $B \subset \mathbb{R}^{n}$,

$$
\begin{aligned}
& \left(\frac{1}{|B|} \int_{B}\left(\sigma\left(u^{\varepsilon}\right)+\varepsilon\right) d x\right)\left(\frac{1}{|B|} \int_{B} \frac{1}{\sigma\left(u^{\varepsilon}\right)+\varepsilon} d x\right) \\
& \quad=\left(\frac{1}{|B|} \int_{B} \sigma\left(u^{\varepsilon}\right) d x\right)\left(\frac{1}{|B|} \int_{B} \frac{1}{\sigma\left(u^{\varepsilon}\right)+\varepsilon} d x\right)+\frac{1}{|B|} \int_{B} \frac{\varepsilon}{\sigma\left(u^{\varepsilon}\right)+\varepsilon} d x \\
& \quad \leqslant\left(\frac{1}{|B|} \int_{B} \sigma\left(u^{\varepsilon}\right) d x\right)\left(\frac{1}{|B|} \int_{B} \frac{1}{\sigma\left(u^{\varepsilon}\right)} d x\right)+1 \\
& \quad \leqslant C
\end{aligned}
$$

which says that $\sigma\left(u^{\varepsilon}\right)+\varepsilon$ is also an $A_{2}$ weight, uniform with respect to time and $\varepsilon$.

Second, we estimate the Hölder norms of $\phi^{\varepsilon}$ and $u^{\varepsilon}$, respectively. In fact, from [11] and [9] we know that any solution of the elliptic equation (23) with the $A_{2}$ coefficient $\sigma\left(u^{\varepsilon}\right)+\varepsilon$ satisfies the Hölder estimates (see Remark 38):

$$
\left\|\phi^{\varepsilon}(\cdot, t)\right\|_{C^{\alpha}(\bar{\Omega})} \leqslant C\left\|h^{\varepsilon}(\cdot, t)\right\|_{C^{\alpha}(\partial \Omega)} \leqslant C \quad \text { for } t \in(0, T), \quad \forall \alpha \in\left(0, \alpha_{0}\right),
$$

for some fixed constant $\alpha_{0} \in(0,1)$.

We proceed to the Hölder estimate of $u^{\varepsilon}$. For any fixed $x_{0} \in \Omega$, we decompose the function $u^{\varepsilon}$ as $u^{\varepsilon}=u_{1}^{\varepsilon}+u_{2}^{\varepsilon}$, where $u_{1}^{\varepsilon}$ and $u_{2}^{\varepsilon}$ are weak solutions of the equations

$$
\begin{cases}\frac{\partial u_{1}^{\varepsilon}}{\partial t}-\nabla \cdot\left(\kappa\left(u^{\varepsilon}\right) \nabla u_{1}^{\varepsilon}\right)=0 & \text { in } \Omega \\ u_{1}^{\varepsilon}=g & \text { on } \partial \Omega \\ u_{1}^{\varepsilon}(x, 0)=u_{0}(x) & \text { for } x \in \Omega\end{cases}
$$

and

$$
\begin{cases}\frac{\partial u_{2}^{\varepsilon}}{\partial t}-\nabla \cdot\left(\kappa\left(u^{\varepsilon}\right) \nabla u_{2}^{\varepsilon}\right)=\nabla \cdot\left[\left(\phi^{\varepsilon}-\phi^{\varepsilon}\left(x_{0}, t\right)\right)\left(\sigma\left(u^{\varepsilon}\right)+\varepsilon\right) \nabla \phi^{\varepsilon}\right] & \text { in } \Omega, \\ u_{2}^{\varepsilon}=0 & \text { on } \partial \Omega, \\ u_{2}^{\varepsilon}(x, 0)=0 & \text { for } x \in \Omega,\end{cases}
$$


respectively. By the De Giorgi-Nash-Moser estimates [20], we have

$$
\left\|u_{1}^{\varepsilon}\right\|_{C^{\alpha, \alpha / 2}\left(\bar{\Omega}_{T}\right)} \leqslant C\left(\|g\|_{C^{\alpha, \alpha / 2}\left(\bar{\Gamma}_{T}\right)}+\left\|u_{0}\right\|_{C^{\alpha}(\bar{\Omega})}\right),
$$

and in order to estimate $\left\|u_{2}^{\varepsilon}\right\|_{C^{\alpha, \alpha / 2}\left(\bar{\Omega}_{T}\right)}$, we set $\vec{f}=\left(\phi^{\varepsilon}-\phi^{\varepsilon}\left(x_{0}, t\right)\right)\left(\sigma\left(u^{\varepsilon}\right)+\varepsilon\right) \nabla \phi^{\varepsilon}$ and apply Propositions 27-30. We see that for $x_{0} \in \Omega, t_{0}>0,0<2 \rho \leqslant R \leqslant$ $\min \left(\operatorname{dist}\left(x_{0}, \partial \Omega\right), \sqrt{t_{0}}\right)$, we have

$$
\begin{aligned}
& \frac{1}{\rho^{n+2+2 \alpha}}\left\|u_{2}^{\varepsilon}-\left(u_{2}^{\varepsilon}\right)_{Q_{\rho}}\right\|_{L^{2}\left(Q_{\rho}\right)}^{2} \\
& \quad \leqslant C\left(\frac{1}{R^{n+2+2 \alpha}}\left\|u_{2}^{\varepsilon}-\theta\right\|_{L^{2}\left(Q_{R}\right)}^{2}+\frac{1}{R^{n+2 \alpha}}\|\vec{f}\|_{L^{2}\left(Q_{R}\right)}^{2}\right), \\
& \quad \leqslant C\left(\frac{1}{R^{n+2+2 \alpha}}\left\|u_{2}^{\varepsilon}-\theta\right\|_{L^{2}\left(Q_{R}\right)}^{2}+\frac{1}{R^{n}}\left\|\phi^{\varepsilon}\right\|_{L^{\infty}\left(I ; C^{\alpha}(\bar{\Omega})\right)}^{2}\left\|\sqrt{\sigma\left(u^{\varepsilon}\right)+\varepsilon} \nabla \phi^{\varepsilon}\right\|_{L^{2}\left(Q_{R}\right)}^{2}\right) \\
& \quad \leqslant C\left(\frac{1}{R^{n+2+2 \alpha}}\left\|u_{2}^{\varepsilon}-\theta\right\|_{L^{2}\left(Q_{R}\right)}^{2}+\frac{1}{R^{n}}\left\|\sqrt{\sigma\left(u^{\varepsilon}\right)+\varepsilon} \nabla \phi^{\varepsilon}\right\|_{L^{2}\left(Q_{R}\right)}^{2}\right) .
\end{aligned}
$$

Similarly, for $x_{0} \in \Omega, t_{0}=0, Q_{R}=B_{R}\left(x_{0}\right) \times\left[0, R^{2}\right]$ and $0<\rho<R \leqslant$ $\min \left(\operatorname{dist}\left(x_{0}, \partial \Omega\right), \sqrt{T}\right)$, we have

$$
\frac{1}{\rho^{n+2+2 \alpha}}\left\|u_{2}^{\varepsilon}\right\|_{L^{2}\left(Q_{\rho}\right)}^{2} \leqslant C\left(\frac{1}{R^{n+2+2 \alpha}}\left\|u_{2}^{\varepsilon}\right\|_{L^{2}\left(Q_{R}\right)}^{2}+\frac{1}{R^{n}}\left\|\sqrt{\sigma\left(u^{\varepsilon}\right)+\varepsilon} \nabla \phi^{\varepsilon}\right\|_{L^{2}\left(Q_{R}\right)}^{2}\right) \text {. }
$$

For $x_{0} \in \partial \Omega, t_{0}>0, Q_{R}=B_{R}\left(x_{0}\right) \cap \Omega \times\left(t_{0}-R^{2}, t_{0}\right]$ and $0<\rho<R \leqslant \operatorname{dist}\left(R_{\Omega}\right.$, $\left.\sqrt{t_{0}}\right)$, we have

$$
\frac{1}{\rho^{n+2+2 \alpha}}\left\|u_{2}^{\varepsilon}\right\|_{L^{2}\left(Q_{\rho}\right)}^{2} \leqslant C\left(\frac{1}{R^{n+2+2 \alpha}}\left\|u_{2}^{\varepsilon}\right\|_{L^{2}\left(Q_{R}\right)}^{2}+\frac{1}{R^{n}}\left\|\sqrt{\sigma\left(u^{\varepsilon}\right)+\varepsilon} \nabla \phi^{\varepsilon}\right\|_{L^{2}\left(Q_{R}\right)}^{2}\right) .
$$

For $x_{0} \in \partial \Omega, t_{0}=0, Q_{R}=B_{R}\left(x_{0}\right) \cap \Omega \times\left[0, R^{2}\right]$ and $0<\rho<R \leqslant \min \left(R_{\Omega}, \sqrt{T}\right)$, we have

$$
\frac{1}{\rho^{n+2+2 \alpha}}\left\|u_{2}^{\varepsilon}\right\|_{L^{2}\left(Q_{\rho}\right)}^{2} \leqslant C\left(\frac{1}{R^{n+2+2 \alpha}}\left\|u_{2}^{\varepsilon}\right\|_{L^{2}\left(Q_{R}\right)}^{2}+\frac{1}{R^{n}}\left\|\sqrt{\sigma\left(u^{\varepsilon}\right)+\varepsilon} \nabla \phi^{\varepsilon}\right\|_{L^{2}\left(Q_{R}\right)}^{2}\right) \text {. }
$$

Combining the last four inequalities and following the outline of Section 4.2, we can derive

$$
\left\|u_{2}^{\varepsilon}\right\|_{\mathcal{L}_{\text {para }}^{2,1+2 \alpha /(n+2)}\left(\Omega_{T}\right)} \leqslant C\left\|\sqrt{\sigma\left(u^{\varepsilon}\right)+\varepsilon} \nabla \phi^{\varepsilon}\right\|_{L_{\text {para }}^{2, n /(n+2)}\left(Q_{R}\right)} .
$$

With (28) and the equivalence relation $\mathcal{L}_{\text {para }}^{2,1+2 \alpha /(n+2)}\left(\Omega_{T}\right) \cong C^{\alpha, \alpha / 2}\left(\bar{\Omega}_{T}\right)$, we see that

$$
\left\|u_{2}^{\varepsilon}\right\|_{C^{\alpha, \alpha / 2}\left(\bar{\Omega}_{T}\right)} \leqslant C
$$


Therefore,

$$
\left\|u^{\varepsilon}\right\|_{C^{\alpha, \alpha / 2}\left(\bar{\Omega}_{T}\right)} \leqslant\left\|u_{1}^{\varepsilon}\right\|_{C^{\alpha, \alpha / 2}\left(\bar{\Omega}_{T}\right)}+\left\|u_{2}^{\varepsilon}\right\|_{C^{\alpha, \alpha / 2}\left(\bar{\Omega}_{T}\right)} \leqslant C .
$$

Third, we present $W^{1, q}$ estimates of $\phi^{\varepsilon}$ and $u^{\varepsilon}$. Note that the last inequality implies that

$$
C^{-1} \leqslant \sigma\left(u^{\varepsilon}\right)+\varepsilon \leqslant C, \quad\left\|\sigma\left(u^{\varepsilon}\right)+\varepsilon\right\|_{C^{\alpha, \alpha / 2}\left(\bar{\Omega}_{T}\right)} \leqslant C, \quad\left\|\kappa\left(u^{\varepsilon}\right)\right\|_{C^{\alpha, \alpha / 2}\left(\bar{\Omega}_{T}\right)} \leqslant C .
$$

With the Hölder estimates of $\sigma\left(u^{\varepsilon}\right)+\varepsilon$ and $\kappa\left(u^{\varepsilon}\right)$, we apply Lemmas 33 and 34 and derive

$$
\begin{aligned}
& \left\|\phi^{\varepsilon}\right\|_{L^{\infty}\left((0, T) ; W^{1, q}(\Omega)\right)} \leqslant C\|h\|_{L^{\infty}\left((0, T) ; W^{1, q}(\Omega)\right)} \leqslant C, \\
& \left\|u^{\varepsilon}\right\|_{L^{p}\left((0, T) ; W^{1, q}(\Omega)\right)} \leqslant C_{p}\left\|\phi^{\varepsilon}\right\|_{L^{p}\left((0, T) ; W^{1, q}(\Omega)\right)}+C_{p} \leqslant C_{p},
\end{aligned}
$$

for some $q>n$ and any $1<p<\infty$. From Equation (22) we also see that

$$
\left\|\partial_{t} u^{\varepsilon}\right\|_{L^{p}\left(I ; W^{-1, q}(\Omega)\right)} \leqslant C\left(\left\|u^{\varepsilon}\right\|_{L^{p}\left((0, T) ; W^{1, q}(\Omega)\right)}+\left\|\nabla \phi^{\varepsilon}\right\|_{L^{p}\left((0, T) ; W^{1, q}(\Omega)\right)}\right) \leqslant C .
$$

Finally, we estimate the interior space-time Hölder norm of $\phi^{\varepsilon}$, which is used to obtain pointwise convergence of the approximating solutions in the next subsection. For the simplicity of notation, we set $A^{\varepsilon}=\sigma\left(u^{\varepsilon}\right)+\varepsilon$. From (23) we see that

$$
\begin{aligned}
& -\nabla \cdot\left(A^{\varepsilon}\left(x, t_{1}\right) \nabla\left[\phi^{\varepsilon}\left(x, t_{1}\right)-\phi^{\varepsilon}\left(x, t_{2}\right)\right]\right) \\
& \quad=\nabla \cdot\left(\left(A^{\varepsilon}\left(x, t_{1}\right)-A^{\varepsilon}\left(x, t_{2}\right)\right) \nabla \phi^{\varepsilon}\left(x, t_{2}\right)\right) .
\end{aligned}
$$

By applying the interior $W^{1, q}$ estimate to the above equation, we find that for any closed ball $\bar{B}_{R}$ contained in $\Omega$ there holds

$$
\begin{aligned}
\left\|\phi^{\varepsilon}\left(x, t_{1}\right)-\phi^{\varepsilon}\left(x, t_{2}\right)\right\|_{L^{\infty}\left((0, T) ; W^{1, q}\left(B_{R}\right)\right)} & \leqslant C_{\operatorname{dist}\left(\bar{B}_{R}, \partial \Omega\right)}\left\|A^{\varepsilon}\left(x, t_{1}\right)-A^{\varepsilon}\left(x, t_{2}\right)\right\|_{L^{\infty}\left(\Omega_{T}\right)} \\
& \leqslant C_{\operatorname{dist}\left(\bar{B}_{R}, \partial \Omega\right)}\left\|A^{\varepsilon}\right\|_{C^{\alpha, \alpha / 2}\left(\bar{\Omega}_{T}\right)}\left|t_{1}-t_{2}\right|^{\alpha / 2},
\end{aligned}
$$

which reduces to

$$
\left\|\phi^{\varepsilon}\right\|_{C^{\alpha / 2}\left([0, T] ; W^{1, q}\left(B_{R}\right)\right)} \leqslant C_{\mathrm{dist}\left(\bar{B}_{R}, \partial \Omega\right)} .
$$

Since $W^{1, q}\left(B_{R}\right) \hookrightarrow C^{\alpha}(\bar{\Omega})$, the last inequality implies that

$$
\left\|\phi^{\varepsilon}\right\|_{C^{\alpha, \alpha / 2}\left(\bar{B}_{R} \times[0, T]\right)} \leqslant C_{\operatorname{dist}\left(\bar{B}_{R}, \partial \Omega\right)} .
$$

The proof of Proposition 37 is complete.

REMARK 38. In (31), we have used both the interior estimate of [11] and a boundary estimate, which is slightly stronger than the result proved in [9, Theorem 4.2]. In fact, from the proof of Theorem 4.2 of [9] one can see that 
there exists $\gamma>0$ such that

$$
\underset{B_{r}\left(x_{0}\right) \cap \Omega}{\operatorname{osc}} \phi^{\varepsilon} \leqslant C\left(\frac{\rho}{R}\right)^{\gamma} \underset{B_{R}\left(x_{0}\right) \cap \Omega}{\operatorname{osc}} \phi^{\varepsilon}+C_{\gamma, \beta}\left\|h^{\varepsilon}\right\|_{C^{\beta}(\partial \Omega)} R^{\beta}
$$

for $x_{0} \in \partial \Omega$ and $\beta<\gamma$. Then an application of Lemma 7 (together with the interior Hölder estimate) yields (31). (If one applies [9, Theorem 4.2] directly, instead of the stronger result used in (31), one still can prove Theorem 2.)

Note that the conditions of [9, Theorem 4.2] are satisfied:

(1) $\sigma\left(u^{\varepsilon}\right)$ is an $A_{2}$ weight:

$$
\left(\frac{1}{|B|} \int_{B} \sigma(u) d x\right)\left(\frac{1}{|B|} \int_{B} \frac{1}{\sigma(u)} d x\right) \leqslant C .
$$

(2) $\sigma\left(u^{\varepsilon}\right)$ has an upper bound in $\Omega$ and is constant in $\mathbb{R}^{n} \backslash \Omega$, which imply that

$$
C^{-1} \int_{B_{R}\left(x_{0}\right)} \sigma\left(u^{\varepsilon}\right) d x \leqslant R^{n} \leqslant C \int_{B_{R}\left(x_{0}\right) \backslash \Omega} \sigma\left(u^{\varepsilon}\right) d x \quad \text { for } x_{0} \in \partial \Omega .
$$

6.3. Existence of solution. Since $C^{\alpha, \alpha / 2}\left(\bar{\Omega}_{T}\right)$ is compactly embedded into $C\left(\bar{\Omega}_{T}\right)$ and $C^{\alpha, \alpha / 2}\left(\bar{B}_{R} \times[0, T]\right)$ is compactly embedded into $C\left(\bar{B}_{R} \times[0, T]\right)$, there exist functions $u \in C^{\alpha, \alpha / 2}\left(\bar{\Omega}_{T}\right), \phi \in L^{\infty}\left(I ; W^{1, q}(\Omega)\right)$ with $\phi \in C^{\alpha, \alpha / 2}\left(\bar{B}_{R} \times[0\right.$, $T]$ ) for any closed ball $\bar{B}_{R}$ contained in $\Omega$, and a sequence $\varepsilon_{k} \rightarrow 0$, such that $u^{\varepsilon_{k}}$ converges to $u$ in the norm of $C\left(\bar{\Omega}_{T}\right), u^{\varepsilon_{k}}$ converges weakly to $u$ in $L^{p}\left(I ; W^{1, q}(\Omega)\right), \partial_{t} u^{\varepsilon_{k}}$ converges weakly to $\partial_{t} u$ in $L^{p}\left(I ; W^{-1, q}(\Omega)\right), \phi^{\varepsilon_{k}}$ converges weakly* to $\phi$ in $L^{\infty}\left(I ; W^{1, q}(\Omega)\right)$, and $\phi^{\varepsilon_{k}}$ converges to $\phi$ pointwise uniformly in each compact subset of $\Omega \times[0, T]$.

From (23) we see that

$$
\int_{\Omega}\left(\sigma\left(u^{\varepsilon_{k}}\right)+\varepsilon_{k}\right) \nabla \phi^{\varepsilon_{k}} \cdot \nabla \varphi d x=0 \quad \text { for any } \varphi \in H_{0}^{1}(\Omega) .
$$

By taking the limit $k \rightarrow \infty$, we obtain

$$
\int_{\Omega} \sigma(u) \nabla \phi \cdot \nabla \varphi d x=0 \quad \text { for any } \varphi \in H_{0}^{1}(\Omega) \text { and a.e. } t \in(0, T) .
$$

Therefore, for any function $v \in C_{0}^{\infty}(\Omega)$,

$$
\begin{aligned}
& \lim _{k \rightarrow \infty} \int_{\Omega} \nabla \cdot\left(\phi^{\varepsilon_{k}}\left(\sigma\left(u^{\varepsilon_{k}}\right)+\varepsilon_{k}\right) \nabla \phi^{\varepsilon_{k}}\right) v d x \\
& \quad=-\lim _{k \rightarrow \infty} \int_{\Omega} \phi^{\varepsilon_{k}}\left(\sigma\left(u^{\varepsilon_{k}}\right)+\varepsilon_{k}\right) \nabla \phi^{\varepsilon_{k}} \cdot \nabla v d x
\end{aligned}
$$




$$
\begin{aligned}
& =-\int_{\Omega} \phi \sigma(u) \nabla \phi \cdot \nabla v d x \\
& =-\int_{\Omega} \sigma(u) \nabla \phi \cdot[\nabla(\phi v)-v \nabla \phi] d x \\
& =\int_{\Omega} \sigma(u)|\nabla \phi|^{2} v d x .
\end{aligned}
$$

From (22) we know that for any $v \in L^{\infty}\left((0, T) ; C_{0}^{\infty}(\Omega)\right)$,

$$
\begin{gathered}
\int_{0}^{T} \int_{\Omega} \frac{\partial u^{\varepsilon_{k}}}{\partial t} v d x d t+\int_{0}^{T} \int_{\Omega} \kappa\left(u^{\varepsilon_{k}}\right)_{\varepsilon_{k}} \nabla u^{\varepsilon_{k}} \cdot \nabla v d x d t \\
\quad=\int_{0}^{T} \int_{\Omega} \nabla \cdot\left(\phi^{\varepsilon_{k}} \frac{1}{\rho\left(u^{\varepsilon_{k}}\right)_{\varepsilon_{k}}} \nabla \phi^{\varepsilon_{k}}\right) v d x d t .
\end{gathered}
$$

By taking the limit $k \rightarrow \infty$, we get

$$
\int_{0}^{T} \int_{\Omega} \frac{\partial u}{\partial t} v d x d t+\int_{0}^{T} \int_{\Omega} \kappa(u) \nabla u \cdot \nabla v d x d t=\int_{0}^{T} \int_{\Omega} \sigma(u)|\nabla \phi|^{2} v d x d t .
$$

From the regularity of $u$ and $\phi$, we know that Equations (38) and (39) actually hold for any $\varphi \in H_{0}^{1}(\Omega)$ and $v \in L^{2}\left((0, T) ; H_{0}^{1}(\Omega)\right)$.

To conclude, we have proved the existence of a weak solution $(u, \phi)$ to Equations (1)-(3) with regularity (9).

6.4. Uniqueness of solution. Suppose that $\left(u_{1}, \phi_{1}\right)$ and $\left(u_{2}, \phi_{2}\right)$ are two pairs of solutions to the initial-boundary value problem (1)-(3), both satisfying (9). Let $\bar{u}=u_{1}-u_{2}$ and $\bar{\phi}=\phi_{1}-\phi_{2}$. Then $\bar{u}$ and $\bar{\phi}$ are weak solutions to the equations

$$
\begin{aligned}
& \frac{\partial \bar{u}}{\partial t}-\nabla \cdot\left(\kappa\left(u_{1}\right) \nabla \bar{u}\right)=\nabla \cdot\left(\left(\kappa\left(u_{1}\right)-\kappa\left(u_{2}\right)\right) \nabla u_{2}\right) \\
& \quad+\left(\sigma\left(u_{1}\right)-\sigma\left(u_{2}\right)\right)\left|\nabla \phi_{1}\right|^{2}+\sigma\left(u_{2}\right) \nabla\left(\phi_{1}+\phi_{2}\right) \cdot \nabla \bar{\phi} \\
& -\nabla \cdot\left(\sigma\left(u_{1}\right) \nabla \bar{\phi}\right)=\nabla \cdot\left(\left(\sigma\left(u_{1}\right)-\sigma\left(u_{2}\right)\right) \nabla \phi_{2}\right),
\end{aligned}
$$

with the following boundary and initial conditions:

$$
\begin{array}{ll}
\bar{u}(x, t)=0, \quad \bar{\phi}(x, t)=0 & \text { for } x \in \partial \Omega, t \in[0, T], \\
\bar{u}(x, 0)=0 & \text { for } x \in \Omega .
\end{array}
$$

For any $\tau \in(0, T)$, we denote $I_{\tau}=(0, \tau)$ and $\Omega_{\tau}=\Omega \times I_{\tau}$. By applying Lemma 35 to parabolic equation (40), we see that for $q>n$ there exists 
$1<p<\infty$ such that

$$
\begin{aligned}
\|\bar{u}\|_{L^{\infty}\left(\Omega_{\tau}\right) \leqslant} & C\left\|\left(\kappa\left(u_{1}\right)-\kappa\left(u_{2}\right)\right) \nabla u_{2}\right\|_{L^{p}\left(I_{\tau} ; L^{q}(\Omega)\right)} \\
& +C\left\|\left(\sigma\left(u_{1}\right)-\sigma\left(u_{2}\right)\right)\left|\nabla \phi_{1}\right|^{2}\right\|_{L^{p}\left(I_{\tau} ; L^{q / 2}(\Omega)\right)} \\
& +C\left\|\sigma\left(u_{2}\right) \nabla\left(\phi_{1}+\phi_{2}\right) \cdot \nabla \bar{\phi}\right\|_{L^{p}\left(I_{\tau} ; L^{q / 2}(\Omega)\right)} \\
\leqslant & C\|\bar{u}\|_{L^{\infty}\left(\Omega_{\tau}\right)}\left(\tau^{1 / 2 p}\left\|\nabla u_{2}\right\|_{L^{2 p}\left(I_{\tau} ; L^{q}(\Omega)\right)}+\left\|\nabla \phi_{1}\right\|_{L^{2 p}\left(I_{\tau} ; L^{q}(\Omega)\right)}^{2}\right) \\
& +C \tau^{1 / 2 p}\left\|\nabla\left(\phi_{1}+\phi_{2}\right)\right\|_{L^{\infty}\left(I_{\tau} ; L^{q}(\Omega)\right)}\|\nabla \bar{\phi}\|_{L^{2 p}\left(I_{\tau} ; L^{q}(\Omega)\right)} \\
\leqslant & C \tau^{1 / 2 p}\|\bar{u}\|_{L^{\infty}\left(\Omega_{\tau}\right)}+C \tau^{1 / 2 p}\|\nabla \bar{\phi}\|_{L^{\infty}\left(I_{\tau} ; L^{q}(\Omega)\right)}
\end{aligned}
$$

where the constant $C$ is independent of $\tau$. With the Hölder regularity of $u_{1}$, by applying the $W^{1, q}$ estimates to (41), we obtain

$$
\|\nabla \bar{\phi}\|_{L^{\infty}\left(I_{\tau} ; L^{q}(\Omega)\right)} \leqslant C\left\|\left(\sigma\left(u_{1}\right)-\sigma\left(u_{2}\right)\right) \nabla \phi_{2}\right\|_{L^{\infty}\left(I_{\tau} ; L^{q}(\Omega)\right)} \leqslant C\|\bar{u}\|_{L^{\infty}\left(\Omega_{\tau}\right)} .
$$

There exists $T_{0}$ such that for $\tau<T_{0}$, the last two inequalities imply that

$$
\|\bar{u}\|_{L^{\infty}\left(\Omega_{\tau}\right)}+\|\nabla \bar{\phi}\|_{L^{\infty}\left(I_{\tau} ; L^{q}(\Omega)\right)}=0 .
$$

By dividing the interval $(0, T)$ into small parts $\left(T_{k}, T_{k+1}\right], k=0,1, \ldots$, each part satisfying $T_{k+1}-T_{k}<T_{0}$, we find that $\bar{u}\left(\cdot, T_{k}\right) \equiv \bar{\phi}\left(\cdot, T_{k}\right) \equiv 0$ implies that $\bar{u}(\cdot, t) \equiv \bar{\phi}(\cdot, t) \equiv 0$ for $t \in\left[T_{k}, T_{k+1}\right]$. This proves the uniqueness of the solution.

\section{Conclusions}

In this paper, we have proved global existence and uniqueness of a weak solution to the degenerate thermistor problem by establishing a uniform-in-time $\overline{\mathrm{BMO}}$ estimate for parabolic equations with possibly discontinuous coefficients. Roughly speaking, $\overline{\mathrm{BMO}}$ is a combination of the conventional BMO in the interior of the domain and $L^{\infty}$ near the boundary. Physical hypotheses (H1) and (H2) are satisfied by metals and many semiconductors. The BMO estimate of parabolic equations established in this paper may also be applied to other equations of mathematical physics.

\section{Acknowledgments}

This work was supported in part by the National Natural Science Foundation of China (NSFC; grant numbers 11301262 and 11401587). The research stay of B. L. at Universität Tübingen was supported by Alexander von Humboldt Foundation.

The authors would like to thank Professor Yanping Lin and Professor Weiwei Sun for the discussions on this topic and the anonymous referees for their valuable comments and suggestions, which were very helpful to improve the presentation of this paper. 


\section{References}

[1] R. A. Adams, Sobolev Spaces, (Academic Press, New York, 1975).

[2] G. Akrivis and S. Larsson, 'Linearly implicit finite element methods for the time dependent joule heating problem', BIT 45 (2005), 429-442.

[3] W. Allegretto and H. Xie, 'Existence of solutions for the time-dependent thermistor equations', IMA J. Appl. Math. 48 (1992), 271-281.

[4] W. Allegretto and N. Yan, 'A posteriori error analysis for FEM of thermistor problems', Int. J. Numer. Anal. Model. 3 (2006), 413-436.

[5] S. N. Antontsev and M. Chipot, 'The thermistor problem: existence, smoothness, uniqueness, blowup', SIAM J. Math. Anal. 25 (1994), 1128-1156.

[6] D. G. Aronson and J. Serrin, 'Local behavior of solutions of quasilinear parabolic equations', Arch. Ration. Mech. Anal. 25 (1967), 81-122.

[7] Y. Z. Chen, Parabolic Partial Differential Equations of Second Order, (Peking University Press, Beijing, 2003), (in Chinese).

[8] G. Cimatti, 'Existence of weak solutions for the nonstationary problem of the joule heating of a conductor', Ann. Mat. Pura Appl. 162 (1992), 33-42.

[9] G. Di Fazio, M. S. Fanciullo and P. Zamboni, 'Harnack inequality and smoothness for quasilinear degenerate elliptic equations', J. Differential Equations 245 (2008), 2939-2957.

[10] C. M. Elliott and S. Larsson, 'A finite element model for the time-dependent joule heating problem', Math. Comp. 64 (1995), 1433-1453.

[11] E. B. Fabes, C. E. Kenig and R. P. Serapioni, 'The local regularity of solutions of degenerate elliptic equations', Comm. Partial Differential Equations 7 (1982), 77-116.

[12] H. Gao, B. Li and W. Sun, 'Unconditionally optimal error estimates of a Crank-Nicolson Galerkin method for the nonlinear thermistor equations', SIAM J. Numer. Anal. 52 (2014), 933-954.

[13] L. Grafakos, Classical and Modern Fourier Analysis, (China Machine Press, Beijing, 2005).

[14] A. E. Hachimi and M. R. S. Ammi, 'Existence of weak solutions for the thermistor problem with degeneracy', Electron. J. Differential Equations (2002), 127-137; (Conference 09).

[15] D. Hömberg, C. Meyer, J. Rehberg and W. Ring, 'Optimal control for the thermistor problem', SIAM J. Control Optim. 48 (2009/10), 3449-3481.

[16] V. Hrynkiv, 'Optimal boundary control for a time dependent thermistor problem', Electron. J. Differential Equations 83 (2009), 1-22.

[17] D. Jerison and C. E. Kenig, 'The inhomogeneous Dirichlet problems in Lipschitz domains', J. Funct. Anal. 130 (1995), 161-219.

[18] K. L. Kuttler, M. Shillor and J. R. Fernández, 'Existence for the thermoviscoelastic thermistor problem', Differ. Equ. Dyn. Syst. 17(3) (2009), 217-233.

[19] B. Li and W. Sun, 'Error analysis of linearized semi-implicit Galerkin finite element methods for nonlinear parabolic equations', Int. J. Numer. Anal. Model. 10 (2013), 622-633.

[20] G. M. Lieberman, Second Order Parabolic Differential Equations, (World Scientific Publishing, Singapore, 1996).

[21] E. D. Macklen, Thermistors, (Electrochemical Publications Ltd, Ayr, 1979).

[22] M. T. G. Montesinos and F. Q. Gallego, 'Existence of a capacity solution to a coupled nonlinear parabolic-elliptic system', Commun. Pure Appl. Anal. 6 (2007), $23-42$.

[23] M. T. G. Montesinos and F. Q. Gallego, 'The evolution thermistor problem under the Wiedemann-Franz law with metallic conduction', Discrete Contin. Dyn. Syst. Ser. B 8 (2007), 901-923.

[24] A. Sommerfeld, Thermodynamics and Statistical Mechanics, (Academic Press, New York, 1964). 
[25] G. M. Troianiello, Elliptic Differential Equations and Obstacle Problems, (Plenum Press, New York, 1987).

[26] I. Wood, 'Maximal $L^{p}$-regularity for the Laplacian on Lipschitz domains', Math. Z. 255 (2007), 855-875.

[27] X. Xu, 'Partial regularity of solutions to a class of degenerate systems', Trans. Amer. Math. Soc. 349 (1997), 1973-1992.

[28] X. Xu, 'On the existence of bounded temperature in the thermistor problem with degeneracy', Nonlinear Anal. 42 (2000), 199-213.

[29] H. Yin, ' $L^{2, \mu}(Q)$-estimates for parabolic equations and applications', IMA Preprint Series [2209], http://purl.umn.edu/2337.

[30] G. Yuan, 'Regularity of solutions of the thermistor problem', Appl. Anal. 53 (1994), $149-156$.

[31] G. Yuan and Z. Liu, 'Existence and uniqueness of the $C^{\alpha}$ solution for the thermistor problem with mixed boundary value', Appl. Anal. 53 (1994), 149-156.

[32] S. Zhou and D. R. Westbrook, 'Numerical solutions of the thermistor equations', J. Comput. Appl. Math. 79 (1997), 101-118.

[33] J. M. Ziman, Electrons and Phonons, (Clarendon Press, Oxford, 1960). 